Linköping Studies in Science and Technology Dissertation No. 1982

\title{
Coherent functors and asymptotic properties
}

\section{Adson Banda}


Linköping Studies in Science and Technology.

Dissertation No. 1982

\title{
Coherent functors and asymptotic properties
}

\author{
Adson Banda
}


Linköping Studies in Science and Technology.

Dissertation No. 1982

Coherent functors and asymptotic properties

Adson Banda

adson. banda@liu.se

www.mai.liu.se

Mathematics and Applied Mathematics

Department of Mathematics

Linköping University

SE-581 83 Linköping

Sweden

ISBN 978-91-7685-095-4ＩSSN 0345-7524

Copyright (C) 2019 Adson Banda

Printed by LiU-Tryck, Linköping, Sweden 2019 
To Maria, Chisomo, Mphaso,

Madaliso and Chimwemwe. 



\section{Abstract}

In this thesis we study properties of the so called coherent functors. Coherent functors were first introduced by Auslander in 1966 in a general setting. Coherent functors have been used since then as powerful tools for different purposes: to describe infinitesimal deformation theory, to describe algebraicity of a stack or to study properties of Rees algebras.

In 1998, Hartshorne proved that half exact coherent functors over a discrete valuation ring $A$ are direct sums of copies of the identity functor, Hom-functors of quotient modules of $A$ and tensor products of quotient modules of $A$. In our first article (Paper A), we obtain a similar characterization for half exact coherent functors over a much wider class of rings: Dedekind domains. This fact allows us to classify half exact coherent functors over Dedekind domains.

In our second article (Paper B), coherent functors over noetherian rings are considered. We study asymptotic properties of sets of prime ideals connected with coherent functors applied to artinian modules or finitely generated modules. Also considering quotient modules $M / \mathfrak{a}^{n} M$, where $\mathfrak{a}^{n}$ is the $n^{\text {th }}$ power of an ideal $\mathfrak{a}$, one obtains that the Betti and Bass numbers of the images under a coherent functor of the quotient modules above are polynomials in $\mathrm{n}$ for large $\mathrm{n}$. Furthermore, the lengths of these image modules are polynomial in $n$, for large $n$, under the condition that the image modules have finite length. 



\section{Populärvetenskaplig sammanfattning}

Denna avhandling behandlar koherenta funktorer på kategorin av moduler över noetherska kommutativa ringar. Koherenta funktorer introducerades allmänt av Auslander 1966. Sedan dess har koherenta funktorer framgångsrikt använts som verktyg i kommutativ algebra och algebraisk geometri. 1998 fann Hartshorne en karakterisering, av så kallade halvexakta koherenta funktorer över vissa ringar: diskreta valuationsringar.

Denna avhandling består av två delar (var del i en artikel): I första delen hittar vi en liknande karakterisering av koherenta funktorer, som den given av Hartshorne, över en bredare klass av ringar: Dedekindområden.

I andra delen studeras asymptotiska egenskaper hos koherenta funktorer på moduler över noetherska ringar. Vi studerar asymptotiska egenskaper när en koherent funktor appliceras på kvotmoduler av en modul genom höga potenser av ideal. 



\section{Acknowledgments}

I would like to express my sincere gratitude to my supervisors, Leif Melkersson and Milagros Izquierdo Barrios, for the thorough supervision in my research. I am grateful for their patient guidance, encouragement, and willingness to give their time so generously towards my research. I am also grateful to them for the useful comments in the write up of this thesis, for advice and also for the administrative work that they have been involved in throughout my studies.

I also acknowledge the funding I have received from the International Science Program (ISP) through Eastern Africa Universities Mathematics Programme (EAUMP) to enable me do my studies. I am thankful to Linköping University and University of Zambia for this opportunity to study.

I also acknowledge my fellow graduate students, present and past, and the members of staff at the Department of Mathematics of Linköping University for providing a conducive environment to do research. I am also grateful to my colleagues at the Department of Mathematics and Statistics of the University of Zambia for covering for me during my absence.

I am forever grateful to my family and friends for the encouragements and the moral and emotional support. I am most grateful to my wife Maria and our children for their patience and endurance. You give me the strength to carry on in my endeavours.

All the persons and organizations not mentioned who in one way or another have been relevant to my studies are hereby acknowledged. 



\section{Contents}

\begin{tabular}{lll}
\hline I Preliminaries and summary of papers & 1
\end{tabular}

1 Category of modules 3

1.1 Categories and Functors $\ldots \ldots \ldots \ldots$

1.2 Abelian Categories $\ldots \ldots \ldots \ldots \ldots \ldots$

1.3 The category of modules $\ldots \ldots \ldots \ldots \ldots$

1.4 Homology functors $\ldots \ldots \ldots \ldots \ldots \ldots \ldots$

1.5 Limits and Completions $\ldots \ldots \ldots \ldots \ldots$

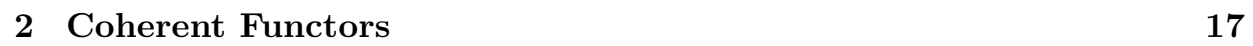

2.1 Coherent Functors $\ldots \ldots \ldots \ldots \ldots \ldots \ldots$

2.2 Left-, right- and half-exact coherent functors $\ldots \ldots \ldots \ldots \ldots$

\begin{tabular}{|lll}
3 & Asymptotic stability & 23
\end{tabular}

3.1 Associated primes ideals . . . . . . . . . . . . . . . 23

3.2 Secondary representations and attached prime ideals . . . . . . . 24

3.3 Asymptotic stability of Ass and Att $\ldots \ldots \ldots \ldots . \ldots . \ldots . \ldots 25$

3.4 Depth and grade of a module $\ldots \ldots \ldots \ldots \ldots \ldots$

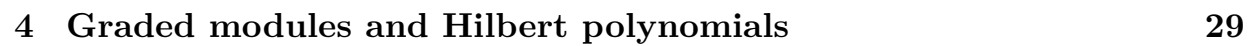

4.1 Length of a module. . . . . . . . . . . . . . . . . . . . . . 29

4.2 Graded and associated graded modules. . . . . . . . . . . . . 30

4.3 Hilbert functions $\ldots \ldots \ldots \ldots \ldots \ldots \ldots \ldots$

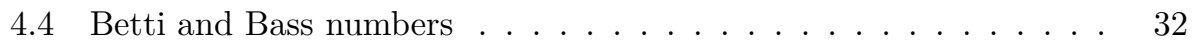

4.5 Betti numbers related to a half exact coherent functor over a DVR 33 
\begin{tabular}{|ll|}
5 & An overview of the papers \\
\hline
\end{tabular}

5.1 Summary of Paper A . . . . . . . . . . . . . . . . . . . . . . . . . . . . 37

5.2 Summary of Paper B . . . . . . . . . . . . . . . . . . 38

\begin{tabular}{|ll}
\hline Bibliography & 41
\end{tabular}

II Included Papers 45

\begin{tabular}{ll}
\hline Paper A & 47
\end{tabular}

\begin{tabular}{ll}
\hline Paper B & 63
\end{tabular} 
Part I

\section{Preliminaries and summary of papers}





\section{1}

\section{Category of modules}

This chapter introduces the relevant categorical notions that are used throughout this thesis. We refer the reader to 13 and 23 for the general perspective, to 10 and [12] for details on abelian categories and to [35] for details on the category of modules.

\subsection{Categories and Functors}

A category $\mathcal{A}$ consists of the following:

(a) objects $A, B, C, D, \ldots$

(b) a set $\operatorname{Hom}_{\mathcal{A}}(A, B)$ of morphisms $f: A \rightarrow B$ between each pair of objects $A, B ;$ and

(c) the law of composition, i.e, given two morphisms $f: A \rightarrow B$ and $g: B \rightarrow C$ in $\mathcal{A}$, the composite $g \circ f$ is defined and satisfies

(i) the associative law

$$
(h \circ g) \circ f=h \circ(g \circ f)
$$

where $h: C \rightarrow D$, and

(ii) the unit law

$$
f \circ 1_{A}=f \text { and } 1_{B} \circ f=f
$$

where $1_{A}: A \rightarrow A$ is the $i d e n t i t y$ morphism.

Note, we say that $f: A \rightarrow B$ is a morphism in $\mathcal{A}$ to mean that $f$ is a morphism in the set $\operatorname{Hom}_{A}(A, B)$ for some pair of objects $A, B$ in $\mathcal{A}$. 
Definition 1.1.1. Given two categories $\mathcal{A}$ and $\mathcal{B}$, we say that $\mathcal{B}$ is

a) a subcategory of $\mathcal{A}$ if

(i) all the objects of $\mathcal{B}$ are also objects of $\mathcal{A}$; and

(ii) every morphism $f$ in $\operatorname{Hom}_{\mathcal{B}}(A, B)$ is also a morphism in $\operatorname{Hom}_{\mathcal{A}}(A, B)$.

b) a full subcategory of $\mathcal{A}$ if, for every pair of objects $B, B^{\prime}$ in $\mathcal{B}$, we have $\operatorname{Hom}_{\mathcal{B}}\left(B, B^{\prime}\right)=\operatorname{Hom}_{\mathcal{A}}\left(B, B^{\prime}\right)$.

Definition 1.1.2. Let $\mathcal{A}$ and $\mathcal{B}$ be categories; a covariant functor $F: \mathcal{A} \rightarrow \mathcal{B}$ with domain $\mathcal{A}$ and co-domain $\mathcal{B}$ sends

(a) each object $A$ in $\mathcal{A}$, to an object $F(A)$ in $\mathcal{B}$; and

(b) each morphism $f: A \rightarrow B$ in $\mathcal{A}$, to a morphism $F(f): F(A) \rightarrow F(B)$ in $\mathcal{B}$ such that the following conditions are satisfied

(i) $F\left(1_{A}\right)=1_{F(A)}$ for every object $A$ in $\mathcal{A}$; and

(ii) $F(g \circ f)=F(g) \circ F(f)$ for all morphisms $f: A \rightarrow B$ and $g: B \rightarrow C$ in $\mathcal{A}$.

The functor that sends every object in $\mathcal{A}$ to itself and every morphism in $\mathcal{A}$ to itself is called the $i d e n t i t y$ functor, and is denoted by $1_{\mathcal{A}}: \mathcal{A} \rightarrow \mathcal{A}$. The composite $G \circ F$ of two functors $F: \mathcal{A} \rightarrow \mathcal{B}$ and $G: \mathcal{B} \rightarrow \mathcal{C}$ is also a functor, and is defined by

(i) $(G \circ F)(A)=G(F(A))$ for every $A$ in $\mathcal{A}$, and

(ii) $(G \circ F)(f)=G(F(f))$ for every morphism $f$ in $\mathcal{A}$.

Note that, given a functor $F: \mathcal{A} \rightarrow \mathcal{B}$ and any pair of objects $A, A^{\prime}$ of $\mathcal{A}$, there is an induced morphism

$$
\Phi_{A, A^{\prime}}: \operatorname{Hom}_{\mathcal{A}}\left(A, A^{\prime}\right) \rightarrow \operatorname{Hom}_{\mathcal{B}}\left(F(A), F\left(A^{\prime}\right)\right)
$$

defined by $\Phi_{A, A^{\prime}}(f)=F(f)$.

Definition 1.1.3. Let $F, G: \mathcal{A} \rightarrow \mathcal{B}$ be functors. A natural transformation $\alpha: F \rightarrow G$ sends each object $A$ in $\mathcal{A}$, to a morphism $\alpha_{A}: F(A) \rightarrow G(A)$ in $\mathcal{B}$ such that the diagram

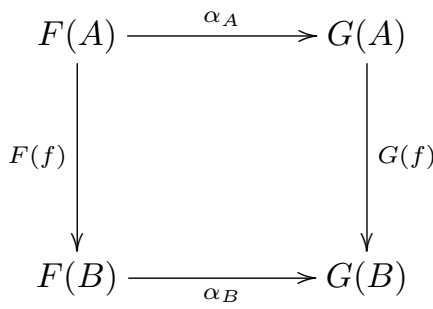

is commutative for every morphism $f: A \rightarrow B$ in $\mathcal{A}$. 
Now, let $F, G, H: \mathcal{A} \rightarrow \mathcal{B}$ be functors and $\alpha: F \rightarrow G$ and $\beta: G \rightarrow H$ be natural transformations. For each object $A$ in $\mathcal{A}$, the composite $\beta \circ \alpha: F \rightarrow H$ is defined by $(\beta \circ \alpha)_{A}=\beta_{A} \circ \alpha_{A}$. That $\beta \circ \alpha$ is a natural transformation follows from the commutative diagram below.

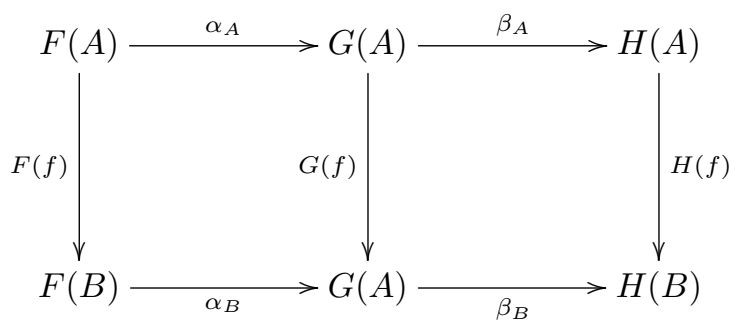

Therefore, the collection of all functors $F: \mathcal{A} \rightarrow \mathcal{B}$ and their natural transformations form the so called functor category.

\subsection{Abelian Categories}

The behavior of abelian categories is similar to that of abelian groups, hence the name.

Definition 1.2.1. Let $A$ and $B$ be objects in $\mathcal{A}$. A product of $A$ and $B$ is an object $A \times B$ in $\mathcal{A}$ together with two morphisms $p_{A}: A \times B \rightarrow A$ and $p_{B}: A \times B \rightarrow B$ such that for any other object $X$ in $\mathcal{A}$ with morphisms $f_{A}: X \rightarrow A$ and $f_{B}: X \rightarrow B$, there exists a unique morphism $f: X \rightarrow A \times B$ making the diagram

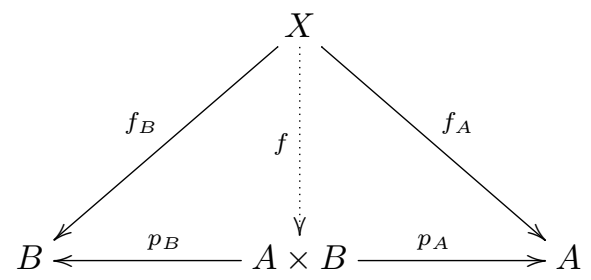

commutative; i.e, $p_{A} \circ f=f_{A}$ and $p_{B} \circ f=f_{B}$.

Definition 1.2.2. Let $A$ and $B$ be objects in $\mathcal{A}$. A coproduct of $A$ and $B$ is an object $A \sqcup B$ in $\mathcal{A}$ together with two morphisms $\iota_{A}: A \rightarrow A \sqcup B$ and $\iota_{B}: B \rightarrow A \sqcup B$ such that for any other object $C$ in $\mathcal{A}$ with morphisms $f_{A}: A \rightarrow C$ and $f_{B}: B \rightarrow C$, there exists a unique morphism $f: A \sqcup B \rightarrow C$ making the diagram

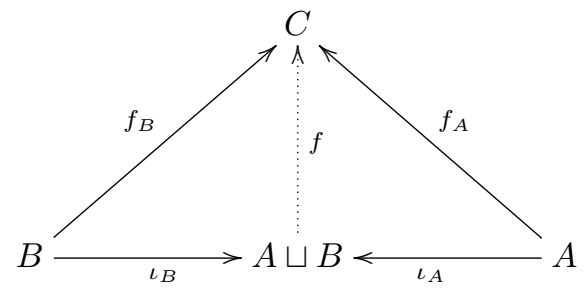


commutative; i.e, $f \circ \iota_{A}=f_{A}$ and $f \circ \iota_{B}=f_{B}$.

In a category $\mathcal{A}$, an object $Z$ such that there are unique morphisms $Z \rightarrow A$ and $A \rightarrow Z$ for each $A$ in $\mathcal{A}$ is called a zero object. A zero morphism is any morphism that factors through a zero object, i.e, if $f: A \rightarrow B$ is a zero morphism, then there exist morphisms $g: A \rightarrow Z$ and $h: Z \rightarrow B$ such that $f=h \circ g$.

Both the zero object and the zero morphism are usually denoted by 0 . The zero object, when it exists, is unique up to isomorphism.

Definition 1.2.3. A category $\mathcal{A}$ is said to be additive if

(i) it has a zero object;

(ii) it has a product $A \times B$ and a coproduct $A \sqcup B$ for each pair of objects $A, B$ in $\mathcal{A}$; and

(iii) its sets of morphisms are abelian groups and composition of morphisms is bilinear, i.e, if $f, f^{\prime} \in \operatorname{Hom}_{\mathcal{A}}(A, B)$ and $g, g^{\prime} \in \operatorname{Hom}_{\mathcal{A}}(B, C)$, then

$$
\begin{gathered}
\left(g+g^{\prime}\right) \circ f=g \circ f+g^{\prime} \circ f \text { and } \\
g \circ\left(f+f^{\prime}\right)=g \circ f+g \circ f^{\prime} .
\end{gathered}
$$

Definition 1.2.4. Let $\mathcal{A}$ be a category with the zero object 0 and let $f: A \rightarrow B$ be a morphism in $\mathcal{A}$.

(i) The kernel of $f$ is a morphism $k: K \rightarrow A$ such that $f \circ k=0$, and such that for every morphism $g: K^{\prime} \rightarrow A$ with $f \circ g=0$ we have $g=k \circ g^{\prime}$ for a unique morphism $g^{\prime}: K^{\prime} \rightarrow K$.

(ii) The cokernel of $f$ is a morphism $c: B \rightarrow C$ such that $c \circ f=0$, and such that for every morphism $h: B \rightarrow C^{\prime}$ with $h \circ f=0$, we have $h=c \circ h^{\prime}$ for a unique morphism $h^{\prime}: C \rightarrow C^{\prime}$.

We denote the kernel of $f$ by $\operatorname{Ker} f$ and the cokernel of $f$ by Coker $f$. It is common practice to just say $K$ is the kernel of $f$ and $C$ is the cokernel of $f$ and we will adopt that.

Definition 1.2.5. Let $f: A \rightarrow B$ be a morphism in any category $\mathcal{A}$. Then, $f$ is said to be

(i) a monomorphism, or a mono, if for every pair of morphisms $g, h: K \rightarrow A$, $f \circ g=f \circ h$ implies $g=h$.

(ii) an epimorphism, or an epi, if for every pair of morphisms $g, h: B \rightarrow C$, $g \circ f=h \circ f$ implies $g=h$.

(iii) an isomorphism if there is another morphism $f^{-1}: B \rightarrow A$ in $\mathcal{A}$ such that $f^{-1} \circ f=1_{A}$ and $f \circ f^{-1}=1_{B}$.

Definition 1.2.6. An abelian category is an additive category $\mathcal{A}$ in which 
(i) every morphism in $\mathcal{A}$ has a kernel and a cokernel; and

(ii) a morphism $f: A \rightarrow B$ is a mono if and only if Ker $f=0$, and it is an epi if and only if Coker $f=0$.

Note that, in an abelian category, a morphism which is both a mono and an epi is an isomorphism.

Definition 1.2.7. Let $\mathcal{A}$ be an abelian category.

(i) An object $P$ in $\mathcal{A}$ is called a projective object if for every morphism $f: P \rightarrow B$ and every epimorphism $g: A \rightarrow B$, there is a morphism $h: P \rightarrow A$ such that $g \circ h=f$.

(ii) We will say that $\mathcal{A}$ has enough projectives if for every object $A$ in $\mathcal{A}$, there is an epimorphism $P \rightarrow A$ where $P$ is a projective object.

(iii) An object $Q \in \mathcal{A}$ is called an injective object if for every morphism $f: A \rightarrow Q$ and every monomorphism $g: A \rightarrow B$, there is a morphism $h: B \rightarrow Q$ such that $f=h \circ g$.

(iv) We will say $\mathcal{A}$ has enough injectives if for every object $A$ in $\mathcal{A}$, there is a monomorphism $A \rightarrow Q$ with $Q$ an injective object.

\subsection{The category of modules}

Of interest to us is the abelian category $\mathcal{M}_{A}$ of $A$-modules and $A$-module homomorphisms and its full subcategory of finitely generated $A$-modules, where $A$ is a noetherian commutative ring with unity. Note that $\mathcal{M}_{A}$ has enough projectives and enough injectives. The interested reader is referred to [31] and [35] for more details.

In this section and also the rest of the thesis, we will write $\operatorname{Hom}_{A}(M, N)$ in place of $\operatorname{Hom}_{\mathcal{M}_{A}}(M, N)$. This section introduces important covariant functors to this thesis, the so called Hom-functors $\operatorname{Hom}_{A}(M,-): \mathcal{M}_{A} \rightarrow \mathcal{M}_{A}$ where $M$ is a fixed $A$-module.

Definition 1.3.1. A functor $F: \mathcal{M}_{A} \rightarrow \mathcal{M}_{A}$ is said to be $A$-linear if, for all pairs $M, N$ of $A$-modules, the induced morphisms

$$
\Phi_{M, N}: \operatorname{Hom}_{A}(M, N) \rightarrow \operatorname{Hom}_{A}(F(M), F(N))
$$

are $A$-module homomorphisms.

We will show that the functor $\operatorname{Hom}_{A}(M,-)$ is a projective object in the category of $A$-linear functors. We first give a description of this functor. Given two homomorphisms $f: M \rightarrow M^{\prime}$ and $g: N \rightarrow N^{\prime}$ in $\mathcal{M}_{A}$, there are homomorphisms

$$
\operatorname{Hom}_{A}(f, N)=\operatorname{Hom}_{A}\left(f, 1_{N}\right): \operatorname{Hom}_{A}\left(M^{\prime}, N\right) \rightarrow \operatorname{Hom}_{A}(M, N)
$$


defined by $h \mapsto h \circ f$ for each homomorphism $h: M^{\prime} \rightarrow N$, and

$$
\operatorname{Hom}_{A}(M, g)=\operatorname{Hom}_{A}\left(1_{M}, g\right): \operatorname{Hom}_{A}(M, N) \rightarrow \operatorname{Hom}_{A}\left(M, N^{\prime}\right)
$$

defined by $h \mapsto g \circ h$ for each homomorphism $h: M \rightarrow N$. Furthermore, the diagram

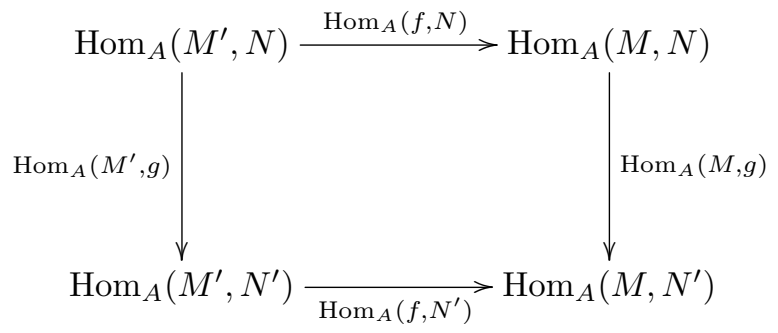

is commutative.

We define the functor $\operatorname{Hom}_{A}(M,-)$ as follows:

(i) $\operatorname{Hom}_{A}(M,-)(N)=\operatorname{Hom}_{A}(M, N)$ for each $A$-module $N$; and

(ii) $\operatorname{Hom}_{A}(M,-)(g)=\operatorname{Hom}_{A}(M, g)$ for each homomorphism $g: N \rightarrow N^{\prime}$. One can show that $\operatorname{Hom}_{A}(M,-)\left(1_{N}\right)=1_{\operatorname{Hom}_{A}(M, N)}$ and that

$$
\begin{aligned}
\operatorname{Hom}_{A}(M,-)(h \circ g) & =\operatorname{Hom}_{A}(M, h) \circ \operatorname{Hom}_{A}(M, g) \\
& =\operatorname{Hom}_{A}(M,-)(h) \circ \operatorname{Hom}_{A}(M,-)(g) .
\end{aligned}
$$

Note that, the commutativity of (1.1) defines a natural transformation

$$
\operatorname{Hom}_{A}(f,-): \operatorname{Hom}_{A}\left(M^{\prime},-\right) \rightarrow \operatorname{Hom}_{A}(M,-)
$$

for each $f: M \rightarrow M^{\prime}$. Also, the functor $\operatorname{Hom}_{A}(A,-)$ is isomorphic to the identity functor on $\mathcal{M}_{A}$.

Definition 1.3.2. Let $M$ be any $A$-module. The $d u a l M^{*}$ of $M$ is defined by $M^{*}=\operatorname{Hom}_{A}(M, A)$.

Note that, for every finitely generated projective $A$-module $P$, the dual $P^{*}$ is also a finitely generated projective $A$-module. Furthermore, we have $P^{* *} \cong P$ for every finitely generated projective $A$-module $P$.

Now, given any $A$-modules $M, L$ and $N$, there is a natural morphism

$$
\Psi: \operatorname{Hom}_{A}(M, L) \otimes_{A} N \rightarrow \operatorname{Hom}_{A}\left(M, L \otimes_{A} N\right)
$$

given by $\left(f \otimes_{A} y\right)(x)=f(x) \otimes_{A} y$ for $f: M \rightarrow L, y \in N$ and $x \in M$. If $M$ is a finitely generated projective $A$-module, $\Psi$ is a natural isomorphism (see Proposition 2 in section 4.2 of Chapter II in [3]). Setting $L=A$ and assuming that $M$ is a finitely generated projective $A$-module in 1.2 , we get a natural isomorphism

$$
M^{*} \otimes_{A} N \cong \operatorname{Hom}_{A}(M, N),
$$


and hence a natural isomorphism

$$
M^{* *} \otimes_{A} N \cong \operatorname{Hom}_{A}\left(M^{*}, N\right) .
$$

Thus, if $P$ is a finitely generated projective $A$-module, we have a natural isomorphism

$$
P \otimes_{A} N \cong \operatorname{Hom}_{A}\left(P^{*}, N\right)
$$

for any $A$-module $N$.

To show that $\operatorname{Hom}_{A}(M,-)$ is a projective object, we need to use the Yoneda lemma which we now state. We refer the reader to Proposition 1.4.3 in [13, pages 61-62 in 23] and Theorem 1.17 in [31] for details and the proof of the lemma.

Theorem 1.3.3 (Yoneda Lemma). Let $F: \mathcal{M}_{A} \rightarrow \mathcal{M}_{A}$ be a covariant functor and $M$ an $A-$ module. There is a bijection:

$$
\operatorname{Nat}\left(\operatorname{Hom}_{A}(M,-), F\right) \cong F(M)
$$

given by $\alpha \mapsto \alpha_{M}\left(1_{M}\right)$ and natural in both $M$ and $F$. Here, $\operatorname{Nat}(G, H)$ denotes the set of natural transformations between the functors $G$ and $H$.

That the functor $\operatorname{Hom}_{A}(M,-)$ is a projective object in the category of all $A$-linear functors on $\mathcal{M}_{A}$ follows from the Yoneda lemma and the commutative diagram

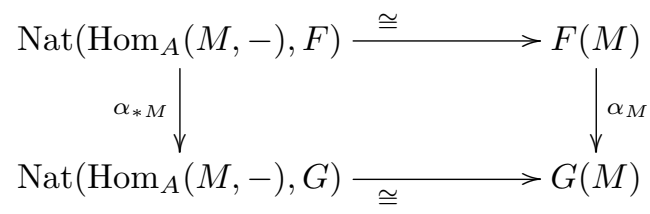

where $\alpha: F \rightarrow G$ is surjective.

We end this section by stating two useful and well known results, namely, the Snake lemma and the Five lemma. Details of these results can be found for instance in [1, [12, [21] and [31.

Theorem 1.3.4 (Snake Lemma). Given a commutative diagram in $\mathcal{M}_{A}$ with exact rows,

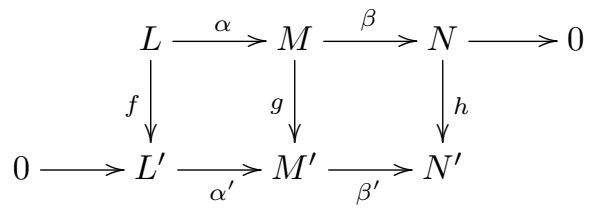

there is a well defined homomorphism $\delta:$ Ker $h \rightarrow$ Coker $f$ called the connecting homomorphism, such that the sequence

Ker $f \stackrel{\sigma}{\longrightarrow}$ Ker $g \stackrel{\tau}{\longrightarrow}$ Ker $h \stackrel{\delta}{\longrightarrow}$ Coker $f \stackrel{\sigma^{\prime}}{\longrightarrow}$ Coker $g \stackrel{\tau^{\prime}}{\longrightarrow}$ Coker $h$ is exact. Furthermore, $\sigma$ is injective if $\alpha$ is injective and $\tau^{\prime}$ is surjective if $\beta^{\prime}$ is surjective. 
Theorem 1.3.5 (Five Lemma). Consider the commutative diagram in $\mathcal{M}_{A}$ with exact rows.

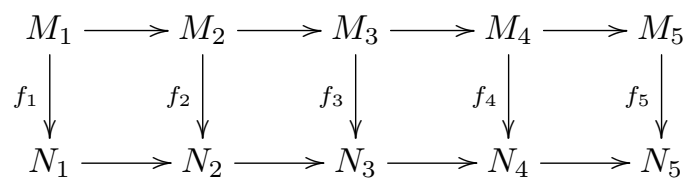

(i) If $f_{2}$ and $f_{4}$ are surjective and $f_{5}$ is injective, then $f_{3}$ is surjective.

(ii) If $f_{2}$ and $f_{4}$ are injective and $f_{1}$ is surjective, then $f_{3}$ is injective.

(iii) If $f_{1}, f_{2}, f_{4}$ and $f_{5}$ are isomorphisms, then $f_{3}$ is an isomorphism.

\subsection{Homology functors}

In this section, we introduce the homology functor $H_{n}(-)$ with values in $\mathcal{M}_{A}$. We refer the reader to [8], 9], [12, [31, [35] and [37] for more details. Throughout this section, $A$ is a commutative noetherian ring.

Definition 1.4.1. A chain complex in $\mathcal{M}_{A}$, denoted by $\mathbf{C}$, is a sequence

$$
\mathbf{C}: \cdots \longrightarrow M_{n+1} \stackrel{d_{n+1}}{\longrightarrow} M_{n} \stackrel{d_{n}}{\longrightarrow} M_{n-1} \longrightarrow \cdots
$$

of modules and homomorphisms in $\mathcal{M}_{A}$ such that $d_{n} \circ d_{n+1}=0$ for all $n \in \mathbb{Z}$.

Definition 1.4.2. Given two chain complexes $\mathbf{C}$ and $\mathbf{C}^{\prime}$ in $\mathcal{M}_{A}$, a chain map $\mathbf{f}: \mathbf{C} \rightarrow \mathbf{C}^{\prime}$ is a sequence of homomorphisms $\left\{f_{n}: M_{n} \rightarrow M_{n}^{\prime}\right\}_{n \in \mathbb{Z}}$ such that the diagram

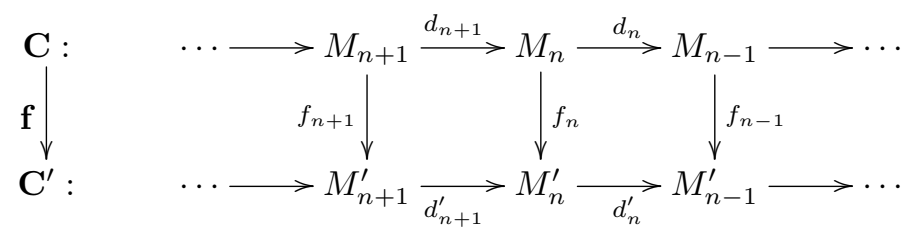

is commutative.

We will denote the identity chain map by $\mathbf{1}_{\mathbf{C}}$. Now, given two chain maps $\mathbf{f}: \mathbf{C} \rightarrow \mathbf{C}^{\prime}$ and $\mathbf{g}: \mathbf{C}^{\prime} \rightarrow \mathbf{C}^{\prime \prime}$, the composite $\mathbf{g} \circ \mathbf{f}: \mathbf{C} \rightarrow \mathbf{C}^{\prime \prime}$ is defined by $(g \circ f)_{n}=g_{n} \circ f_{n}$ for all $n \in \mathbb{Z}$. The chain complexes in $\mathcal{M}_{A}$ form an abelian category; see Proposition 5.100 in 31] for details.

Definition 1.4.3. A chain complex

$$
\mathbf{C}: \cdots \longrightarrow M_{n+1} \stackrel{d_{n+1}}{\longrightarrow} M_{n} \stackrel{d_{n}}{\longrightarrow} M_{n-1} \longrightarrow \cdots
$$

is exact if $\operatorname{Im} d_{n+1}=\operatorname{Ker} d_{n}$ for all $n \in \mathbb{Z}$. 


\section{Example 1.1}

1. Every morphism $f: M_{1} \rightarrow M_{0}$ of $A$-modules gives rise to a complex

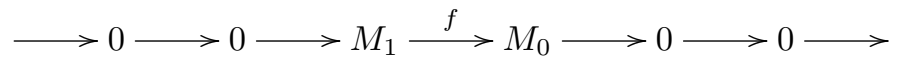

where $M_{n}=0$ for $n>1$ and $M_{n}=0$ for $n<0$.

2. Similarly every short exact sequence

$$
0 \longrightarrow M_{2} \stackrel{f}{\longrightarrow} M_{1} \stackrel{g}{\longrightarrow} M_{0} \longrightarrow 0
$$

gives rise to a complex

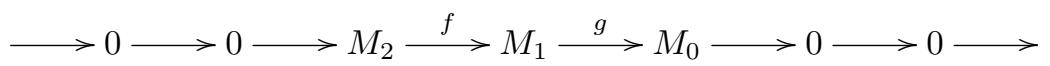

An exact sequence

$$
\mathbf{P}: \cdots \longrightarrow P_{2} \stackrel{d_{2}}{\longrightarrow} P_{1} \stackrel{d_{1}}{\longrightarrow} P_{0} \stackrel{f}{\longrightarrow} M \longrightarrow 0
$$

in $\mathcal{M}_{A}$ is called a projective resolution of an $A$-module $M$ if each $P_{n}$ is a projective $A$-module for all $n=0,1,2, \ldots$ If $(1.3)$ is a projective resolution for $M$, a deleted projective resolution of $M$ is the chain complex

$$
\mathbf{P}_{M}: \cdots \stackrel{d_{3}}{\longrightarrow} P_{2} \stackrel{d_{2}}{\longrightarrow} P_{1} \stackrel{d_{1}}{\longrightarrow} P_{0} \longrightarrow 0
$$

in $\mathcal{M}_{A}$.

Definition 1.4.4. Let

$$
\mathbf{C}: \cdots \longrightarrow M_{n+1} \stackrel{d_{n+1}}{\longrightarrow} M_{n} \stackrel{d_{n}}{\longrightarrow} M_{n-1} \longrightarrow \cdots
$$

be a chain complex in $\mathcal{M}_{A}, Z_{n}(\mathbf{C})=\operatorname{Ker} d_{n}$ and $B_{n}(\mathbf{C})=\operatorname{Im} d_{n+1}$. Then, for $n \in \mathbb{Z}$, the $n^{\text {th }}$ homology $H_{n}(-)$ is defined on the complex $\mathbf{C}$ by

$$
H_{n}(\mathbf{C})=Z_{n}(\mathbf{C}) / B_{n}(\mathbf{C}) .
$$

Since $\operatorname{Im} d_{n+1} \subseteq \operatorname{Ker} d_{n}$, we have an exact sequence

$$
0 \rightarrow B_{n}(\mathbf{C}) \rightarrow Z_{n}(\mathbf{C}) \rightarrow H_{n}(\mathbf{C}) \rightarrow 0
$$

for each $n$. Furthermore, a chain complex $\mathbf{C}$ is exact if and only if $H_{n}(\mathbf{C})=0$ for all $n$. The $n^{\text {th }}$ homology $H_{n}(-)$ is a functor from the category of complexes to $\mathcal{M}_{A}$ for all $n \in \mathbb{Z}$, see for instance Proposition 6.8 in 31 for details.

Now, let $F: \mathcal{M}_{A} \rightarrow \mathcal{M}_{A}$ be a covariant functor and let

$$
\mathbf{C}: \cdots \longrightarrow M_{n+1} \stackrel{d_{n+1}}{\longrightarrow} M_{n} \stackrel{d_{n}}{\longrightarrow} M_{n-1} \longrightarrow \cdots
$$


be a chain complex in $\mathcal{M}_{A}$. Then $F(\mathbf{C})$ :

$$
\cdots \longrightarrow F\left(M_{n+1}\right) \stackrel{F\left(d_{n+1}\right)}{\longrightarrow} F\left(M_{n}\right) \stackrel{F\left(d_{n}\right)}{\longrightarrow} F\left(M_{n-1}\right) \longrightarrow \cdots
$$

is also a chain complex since $F\left(d_{n}\right) \circ F\left(d_{n+1}\right)=F\left(d_{n} \circ d_{n+1}\right)=F(0)=0$ for all $n \in \mathbb{Z}$. For example, consider a chain complex

$$
\mathbf{C}: \cdots \longrightarrow M_{n+1} \stackrel{d_{n+1}}{\longrightarrow} M_{n} \stackrel{d_{n}}{\longrightarrow} M_{n-1} \longrightarrow \cdots
$$

in $\mathcal{M}_{A}$ and the covariant functor $-\otimes_{A} N$ for any $A$-module $N$. We get the chain complex $\mathbf{C} \otimes_{A} N$ :

$$
\cdots \rightarrow M_{n+1} \otimes_{A} N \stackrel{d_{n+1} \otimes_{A} 1_{N}}{\longrightarrow} M_{n} \otimes_{A} N \stackrel{d_{n} \otimes_{A} 1_{N}}{\longrightarrow} M_{n-1} \otimes_{A} N \rightarrow \cdots
$$

and hence we can define, for all $n \in \mathbb{Z}$, the homology functor $H_{n}\left(\mathbf{C} \otimes_{A}-\right)$ by

$$
H_{n}\left(\mathbf{C} \otimes_{A} N\right)=\operatorname{Ker}\left(d_{n} \otimes_{A} 1_{N}\right) / \operatorname{Im}\left(d_{n+1} \otimes_{A} 1_{N}\right) .
$$

\subsection{Limits and Completions}

In this section, $A$ is a commutative ring, $\mathcal{M}_{A}$ is the category of $A$-modules and $I$ is a directed set; i.e, a nonempty partially ordered set such that, for each pair $i, j \in I$, there is $k \in I$ with $i \leq k$ and $j \leq k$.

Definition 1.5.1. A direct system in $\mathcal{M}_{A}$ is an ordered pair $\left\{M_{i}, \varphi_{j}^{i}\right\}_{i \leq j}$ where

(i) $\left\{M_{i}\right\}_{i \in I}$ is a family of $A$-modules, and

(ii) $\left\{\varphi_{j}^{i}: M_{i} \rightarrow M_{j}\right\}_{i \leq j}$ is a family of homomorphisms with $\varphi_{i}^{i}=1_{M_{i}}$ and such that, for $i \leq j \leq k$, the diagram

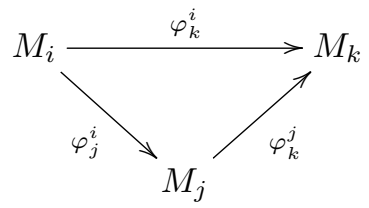

is commutative.

Definition 1.5.2. A direct limit of the direct system $\left\{M_{i}, \varphi_{j}^{i}\right\}_{i \leq j}$ is an $A$-module $\stackrel{\lim }{\longrightarrow} M_{i}$ and a family of homomorphisms $\left\{q_{i}: M_{i} \rightarrow \underset{\lim }{\longrightarrow} M_{i}\right\}_{i \in I}$ such that

(i) $q_{j} \circ \varphi_{j}^{i}=q_{i}$ for all $i \leq j$, and

(ii) for all A-modules $X$ and each family of homomorphisms $\left\{g_{i}: M_{i} \rightarrow X\right\}_{i \in I}$ such that $g_{j} \circ \varphi_{j}^{i}=g_{i}$ for all $i \leq j$, there is a unique module homomorphism 
$\theta: \lim _{\longrightarrow} M_{i} \rightarrow X$ making the diagram

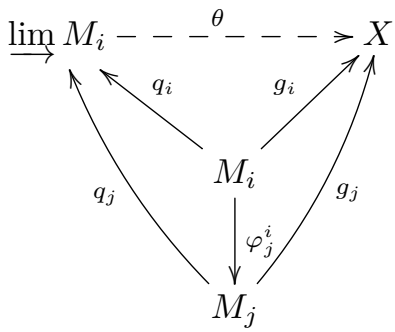

commutative.

Definition 1.5.3. An inverse system in $\mathcal{M}_{A}$ is an ordered pair $\left\{M_{i}, \psi_{i}^{j}\right\}_{i \leq j}$ where

(i) $\left\{M_{i}\right\}_{i \in I}$ is a family of $A$-modules, and

(ii) $\left\{\psi_{i}^{j}: M_{j} \rightarrow M_{i}\right\}_{i \leq j}$ is a family of homomorphisms with $\psi_{i}^{i}=1_{M_{i}}$ and such that, for $i \leq j \leq k$, the diagram

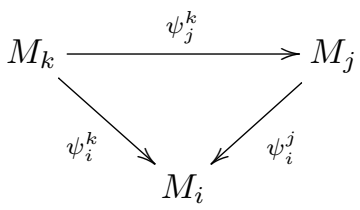

is commutative.

Definition 1.5.4. An inverse limit of an inverse system $\left\{M_{i}, \psi_{i}^{j}\right\}_{i \leq j}$ is an $A$ module $\lim _{\longleftarrow} M_{i}$ and a family $\left\{p_{i}: \lim M_{i} \rightarrow M_{i}\right\}_{i \in I}$ of homomorphisms such that

(i) $\psi_{i}^{j} \circ p_{j}=p_{i}$ for all $i \leq j$, and

(ii) for all A-modules $X$ and each family of homomorphisms $\left\{f_{i}: X \rightarrow M_{i}\right\}_{i \in I}$ such that $\psi_{i}^{j} \circ f_{j}=f_{i}$ for all $i \leq j$, there is a unique module homomorphism $\phi: X \rightarrow \underset{\lim }{\longleftarrow} M_{i}$ making the diagram

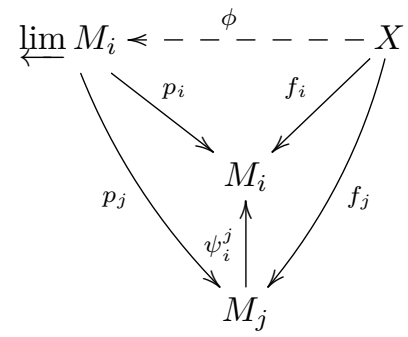

commutative. 
Given an inverse system $\left\{M_{i}, \psi_{i}^{j}\right\}_{i \leq j}$ in $\mathcal{M}_{A}$, there is a natural isomorphism

$$
\operatorname{Hom}_{A}\left(X, \lim _{\longleftarrow} M_{i}\right) \cong \lim _{\longleftarrow} \operatorname{Hom}_{A}\left(X, M_{i}\right)
$$

for every $A-$ module $X$, see Proposition 5.21 in [31].

Definition 1.5.5. A surjective system in $\mathcal{M}_{A}$ is an inverse system $\left\{M_{i}, \psi_{i}^{j}\right\}_{i \leq j}$ in which each homomorphism $\psi_{i}^{j}: M_{j} \rightarrow M_{i}$ is surjective.

It is common practice to denote the inverse system $\left\{M_{i}, \psi_{i}^{j}\right\}_{i \leq j}$ by $\left\{M_{i}\right\}$ and we will use this notation from now onward.

Definition 1.5.6. Let $\left\{M_{i}\right\}$ and $\left\{N_{i}\right\}$ be two inverse systems (both indexed by $I$ ) in $\mathcal{M}_{A}$. A homomorphism $f:\left\{M_{i}\right\} \rightarrow\left\{N_{i}\right\}$ is a family of homomorphisms $\left\{f_{i}: M_{i} \rightarrow N_{i}\right\}$ such that the diagram

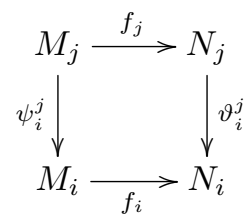

is commutative.

We say that a sequence of inverse systems

$$
0 \rightarrow\left\{X_{i}\right\} \rightarrow\left\{Y_{i}\right\} \rightarrow\left\{Z_{i}\right\} \rightarrow 0
$$

is exact if, for each $i$, the sequence

$$
0 \rightarrow X_{i} \rightarrow Y_{i} \rightarrow Z_{i} \rightarrow 0
$$

is exact. Now, given an exact sequence of inverse systems

$$
0 \rightarrow\left\{X_{i}\right\} \rightarrow\left\{Y_{i}\right\} \rightarrow\left\{Z_{i}\right\} \rightarrow 0
$$

the sequence

$$
0 \rightarrow \lim _{\longleftarrow} X_{i} \rightarrow \lim _{\longleftarrow} Y_{i} \rightarrow \lim _{\longleftarrow} Z_{i}
$$

is always exact, see Proposition 10.2 in [1] for details.

Definition 1.5.7. Let $I=\mathbb{N}$ be the set of positive integers. An inverse system $\left\{M_{i}\right\}$ in $\mathcal{M}_{A}$ is said to satisfy the Mittag-Lefler condition if, for each $i$, the decreasing sequence $\left\{\psi_{i}^{j}\left(M_{j}\right)\right\}_{i \leq j}$ becomes constant for sufficiently large $j$.

The Mittag-Lefler condition is obviously satisfied when $\left\{M_{i}\right\}$ is a surjective system. Furthermore, if

$$
0 \rightarrow\left\{X_{i}\right\} \rightarrow\left\{Y_{i}\right\} \rightarrow\left\{Z_{i}\right\} \rightarrow 0
$$


is an exact sequence of inverse systems and $\left\{X_{i}\right\}$ satisfies the Mittag-Lefler condition, then the sequence

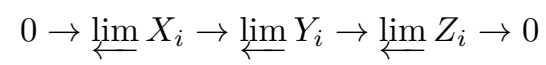

is also exact, see Proposition 10.3 in [21] for details.

Now, for any proper ideal $\mathfrak{a}$ in $A$ and any $A$-module $M$, we have a descending sequence of submodules

$$
M \supseteq \mathfrak{a} M \supseteq \mathfrak{a}^{2} M \supseteq \mathfrak{a}^{3} M \supseteq \ldots
$$

where $\mathfrak{a}^{0}=A$. For $m \geq n$, the inclusions $\mathfrak{a}^{m} M \subseteq \mathfrak{a}^{n} M$ induce a family of homomorphisms

$$
\left\{\psi_{m}^{n}: M / \mathfrak{a}^{m} M \rightarrow M / \mathfrak{a}^{n} M\right\} .
$$

Thus, $\left\{M / \mathfrak{a}^{n} M\right\}$ is a surjective inverse system in $\mathcal{M}_{A}$. The inverse limit of this system is called the $\mathfrak{a}$-adic completion and is denoted by $\widehat{M}$, i.e, $\widehat{M}=\lim _{\longleftarrow} M / \mathfrak{a}^{n} M$. If $M=A, \hat{A}$ is said to be the completion ring of $A$ and for each $A$-module $M, \widehat{M}$ is an $\hat{A}$-module.

If $A$ is noetherian and $M$ is finitely generated, then $\hat{A} \otimes_{A} M \cong \widehat{M}$. Furthermore, given an exact sequence

$$
0 \rightarrow M^{\prime} \rightarrow M \rightarrow M^{\prime \prime} \rightarrow 0
$$

of finitely generated $A$-modules, the sequence

$$
0 \rightarrow \widehat{M}^{\prime} \rightarrow \widehat{M} \rightarrow \widehat{M}^{\prime \prime} \rightarrow 0
$$

is exact. 



\section{Coherent Functors}

Most of the material we present in this chapter is found in [2] and [11]. Throughout this chapter, $A$ is a commutative noetherian ring, $\mathcal{M}_{A}$ is the category of $A$-modules and all functors are $A$-linear on $M_{A}$.

\subsection{Coherent Functors}

Definition 2.1.1. An $A$-linear functor $F$ is said to be coherent if there is an exact sequence

$$
\mathrm{h}_{N} \rightarrow \mathrm{h}_{M} \rightarrow F \rightarrow 0
$$

where $M, N$ are finitely generated $A$-modules. Here, $\mathrm{h}_{X}$ denotes the covariant functor $\operatorname{Hom}_{A}(X,-)$, where $X$ is a finitely generated $A$-module.

Obviously, coherent functors send finitely generated $A$-modules to finitely generated $A$-modules. Note also that, the morphism $\mathrm{h}_{N} \rightarrow \mathrm{h}_{M}$ is induced by a unique module homomorphism $f: M \rightarrow N$. However, the choice of the exact sequence 2.1 is not unique.

Also note that the category of coherent functors has enough projectives since $\mathrm{h}_{X}$ is a projective object in the category of $A$-linear functors (see section 1.3 ). Setting $C=\operatorname{Coker}(f: M \rightarrow N)$, we obtain an exact sequence

$$
0 \rightarrow \mathrm{h}_{C} \rightarrow \mathrm{h}_{N} \rightarrow \mathrm{h}_{M} \rightarrow F \rightarrow 0 .
$$

Coherent functors have, among others, the following properties:

(i) Given an exact sequence $0 \rightarrow F \rightarrow H \rightarrow G \rightarrow 0$ of $A$-linear functors where $F$ and $G$ are coherent, then $H$ is also coherent. 
(ii) If $\alpha: F \rightarrow G$ is a natural transformation between coherent functors, then $\operatorname{Ker} \alpha, \operatorname{Im} \alpha$ and Coker $\alpha$ are also coherent.

(iii) Coherent functors preserve direct limits: Let $\left\{X_{i}\right\}$ be a direct system and consider the commutative diagram.

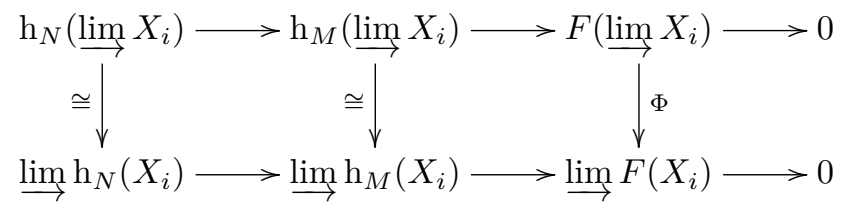

By 1.3.5 the induced map $\Phi$ is an isomorphism .

We refer the reader to [2] or [1] for proofs of properties (i) and (ii). We now give some 'well known' examples of coherent functors.

\section{Example 2.1}

1. Let $M$ be a finitely generated $A$-module. The functor $h_{M}=\operatorname{Hom}_{A}(M,-)$ is coherent since the sequence

$$
\mathrm{h}_{0} \rightarrow \mathrm{h}_{M} \rightarrow \mathrm{h}_{M} \rightarrow 0
$$

is exact.

2. Let $P_{1} \rightarrow P_{2} \rightarrow M \rightarrow 0$ be a projective resolution of $M$ in $\mathcal{M}_{A}$ with each $P_{i}, i=1,2$, finitely generated. Due to the natural isomorphism

$$
P \otimes_{A} N \cong \operatorname{Hom}_{A}\left(P^{*}, N\right)
$$

(see section 1.3), the sequence

$$
\mathrm{h}_{P_{1}^{*}} \rightarrow \mathrm{h}_{P_{2}^{*}} \rightarrow M \otimes_{A}-\rightarrow 0
$$

is exact. Thus, $M \otimes-$ is a coherent functor.

3. Let $\mathbf{P}: \ldots P_{i+1} \rightarrow P_{i} \rightarrow P_{i-1} \rightarrow \ldots$ be a complex in $\mathcal{M}_{A}$, where for each $i$, $P_{i}$ is finitely generated. Consider the chain complex

$$
\mathbf{P} \otimes_{A}-: \ldots \longrightarrow P_{i+1} \otimes_{A}-\stackrel{\alpha}{\longrightarrow} P_{i} \otimes_{A}-\stackrel{\beta}{\longrightarrow} P_{i-1} \otimes_{A}-\longrightarrow \cdots
$$

Let $B_{i}(-)=\operatorname{Im} \alpha$ and $Z_{i}(-)=\operatorname{Ker} \beta$. Then, $Z_{i}(-)$ and $B_{i}(-)$ are coherent functors by property (ii) above. Since

$$
0 \rightarrow B_{i}(-) \rightarrow Z_{i}(-) \rightarrow H_{i}(\mathbf{P} \otimes-) \rightarrow 0
$$

is exact, again by property (ii), the homology functor $H_{i}\left(\mathbf{P} \otimes_{A}-\right)$ is coherent for each $i$. In particular, for any finitely generated $A$-module $M$, the functor $\operatorname{Tor}_{i}^{A}(M,-)$ defined on each $A$-module $N$ by $\operatorname{Tor}_{i}^{A}(M, N)=H_{i}\left(\mathbf{P}_{M} \otimes_{A} N\right)$ is coherent for each $i$. Here, $\mathbf{P}_{M}$ is a deleted projective resolution for $M$. 
4. Similarly, the cohomology functor $H^{i}\left(\operatorname{Hom}_{A}(\mathbf{P},-)\right)$ is coherent for each $i$. In particular, for any finitely generated $A$-module $M$, the functor $\operatorname{Ext}_{A}^{i}(M,-)$ defined on each $A$-module $N$ by $\operatorname{Ext}_{A}^{i}(M, N)=H^{i}\left(\operatorname{Hom}_{A}\left(\mathbf{P}_{M}, N\right)\right)$ is coherent for each $i$.

\subsection{Left-, right- and half-exact coherent functors}

An $A$-linear functor $F$ is said to be

(i) exact if $0 \rightarrow F\left(M^{\prime}\right) \rightarrow F(M) \rightarrow F\left(M^{\prime \prime}\right) \rightarrow 0$ is exact,

(ii) half exact if $F\left(M^{\prime}\right) \rightarrow F(M) \rightarrow F\left(M^{\prime \prime}\right)$ is exact,

(iii) left exact if $0 \rightarrow F\left(M^{\prime}\right) \rightarrow F(M) \rightarrow F\left(M^{\prime \prime}\right)$ is exact,

(iv) right exact if $F\left(M^{\prime}\right) \rightarrow F(M) \rightarrow F\left(M^{\prime \prime}\right) \rightarrow 0$ is exact,

whenever $0 \rightarrow M^{\prime} \rightarrow M \rightarrow M^{\prime \prime} \rightarrow 0$ is exact in $\mathcal{M}_{A}$.

Let $F$ be any $A$-linear functor and define a natural transformation

$$
\alpha: F(A) \otimes_{A}-\rightarrow F
$$

as follows: From $\mu_{m}: A \rightarrow M$, given by $\mu_{m}(a)=a m$, we have the morphism $F\left(\mu_{m}\right): F(A) \rightarrow F(M)$. For any $A$-module $M$, define $\alpha_{M}: F(A) \otimes M \rightarrow F(M)$ by $\alpha_{M}(\xi \otimes m)=F\left(\mu_{m}\right)(\xi)$.

We therefore have an exact sequence

$$
F(A) \otimes-\frac{\alpha}{\longrightarrow} F \longrightarrow F_{0} \longrightarrow 0
$$

where $F_{0}=$ Coker $\alpha$. Since $\alpha_{A}$ is an isomorphism by definition, we have $F_{0}(A)=0$. Also, $F_{0}$ will be coherent whenever $F$ is coherent.

It is clear from 2.2 that $F$ is right exact if $\alpha$ is an isomorphism. Conversely, suppose that $F$ is right exact. Since $\alpha_{A}$ is an isomorphism, for any free $A$-module $L, \alpha_{L}$ is also an isomorphism. Let $L_{0}$ and $L_{1}$ be free $A$-modules such that

$$
L_{1} \rightarrow L_{0} \rightarrow M \rightarrow 0
$$

is exact. Consider the commutative diagram

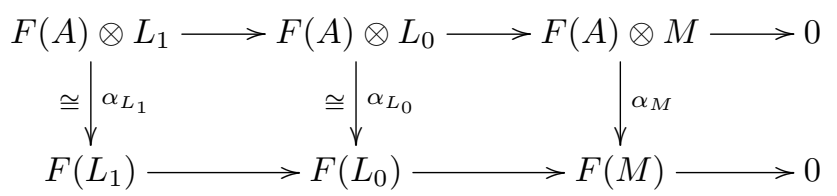

It follows by 1.3 .5 that $\alpha_{M}$ is an isomorphism. 
Suppose now that $F$ is half exact, and let

$$
0 \rightarrow M^{\prime} \rightarrow M \rightarrow M^{\prime \prime} \rightarrow 0
$$

be an exact sequence in $\mathcal{M}_{A}$. Consider the commutative diagram

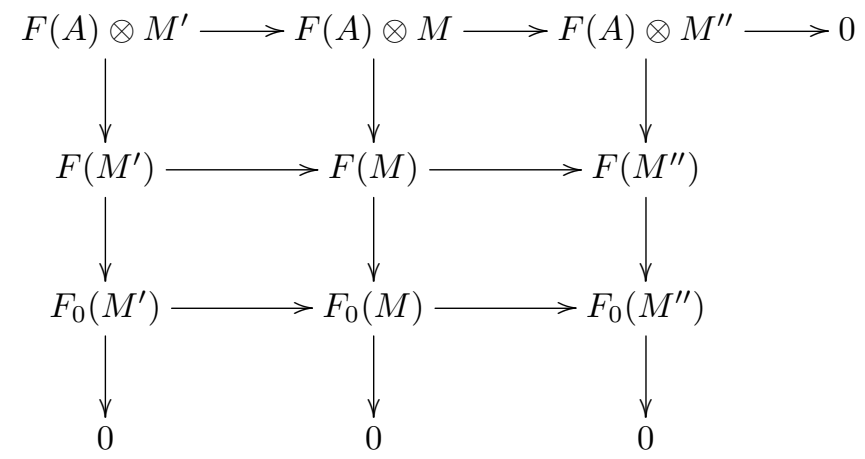

A diagram chase shows that $F_{0}$ is half exact.

We now state a result that has been useful in proving our main results in our first paper.

Theorem 2.2.1. [11, Proposition 3.12] Given that $F$ is a coherent functor, there exists a finitely generated $A$-module $X$, unique up to isomorphism, and a natural transformation $\beta: F \rightarrow \mathrm{h}_{X}$ such that

(i) $\beta_{Q}$ is an isomorphism for every injective $A-$ module $Q$. Hence, if $F_{1}=\operatorname{Ker} \beta$, then $F_{1}(Q)=0$ whenever $Q$ is an injective $A$-module

(ii) $F$ is left exact if and only if $\beta$ is an isomorphism. In, particular, $F$ is left exact if and only if $F \cong \mathrm{h}_{X}$ for some finitely generated $A$-module $X$.

Corollary 2.2.2. Left exact coherent functors preserve inverse limits of directed sets.

Proof. Since every left exact coherent functor is isomorphic to $\mathrm{h}_{N}$ for some finitely generated module $N$, it preserves inverse limits by 1.5 .

Definition 2.2.3. An $A$-module $C$ is said to be a cogenerator of $\mathcal{M}_{A}$ if, for every $A$-module $M$ and every nonzero element $m$ in $M$, there exists a homomorphism $h: M \rightarrow C$ with $h(m) \neq 0$.

Definition 2.2.4. An injective $A$-module $Q$ is said to be an injective hull (or injective envelope) of an $A$-module $M$, denoted by $\operatorname{Env}(M)$, if $M \subset Q$ and there is no injective $A$-module $Q^{\prime}$ such that $M \subset Q^{\prime} \subsetneq Q$.

Proposition 2.2.5. For any coherent functor $F$, the following are equivalent:

(i) $F(C)=0$ when $C$ is an injective cogenerator of $\mathcal{M}_{A}$.

(ii) $F(\operatorname{Env}(A / \mathfrak{m}))=0$, where $\mathfrak{m}$ is a maximal ideal of $A$. 
(iii) Let $M$ and $N$ be finitely generated $A$-modules. Every $f: M \rightarrow N$ such that $\mathrm{h}_{N} \rightarrow \mathrm{h}_{M} \rightarrow F \rightarrow 0$ is exact, must be an injective homomorphism.

(iv) Let $M$ and $N$ be finitely generated $A$-modules. There is an injective homomorphism $f: M \rightarrow N$ such that $\mathrm{h}_{N} \rightarrow \mathrm{h}_{M} \rightarrow F \rightarrow 0$ is exact.

Proof. $\quad(\mathrm{i}) \Rightarrow$ (ii): Since $A / \mathfrak{m}$ is simple for every maximal ideal $\mathfrak{m}$ of $A$, by Theorem 19.8 in [20], the cogenerator $C$ of $\mathcal{M}_{A}$ contains $\operatorname{Env}(A / \mathfrak{m})$ as a direct summand. Hence, $F(\operatorname{Env}(A / \mathfrak{m}))=0$ since $F$ preserve direct summands.

(ii) $\Rightarrow$ (iii): Let $M$ and $N$ be finitely generated $A$-modules and let $f: M \rightarrow N$ be a homomorphism such that $\mathrm{h}_{N} \rightarrow \mathrm{h}_{M} \rightarrow F \rightarrow 0$ is exact. Since by hypothesis $F(\operatorname{Env}(A / \mathfrak{m}))=0$,

$$
\mathrm{h}_{N}(\operatorname{Env}(A / \mathfrak{m})) \rightarrow \mathrm{h}_{M}(\operatorname{Env}(A / \mathfrak{m})) \rightarrow 0
$$

is exact. Now, if $K=\operatorname{Ker} f$, we also have an exact sequence

$$
\mathrm{h}_{N}(\operatorname{Env}(A / \mathfrak{m})) \rightarrow \mathrm{h}_{M}(\operatorname{Env}(A / \mathfrak{m})) \rightarrow \mathrm{h}_{K}(\operatorname{Env}(A / \mathfrak{m})) \rightarrow 0 .
$$

This shows that $\mathrm{h}_{K}(\operatorname{Env}(A / \mathfrak{m}))=\operatorname{Hom}_{A}(K, \operatorname{Env}(A / \mathfrak{m}))=0$. Thus for all maximal ideals $\mathfrak{m}$ is $A$, we have $\operatorname{Hom}_{A_{\mathfrak{m}}}\left(K_{\mathfrak{m}}, \operatorname{Env}(A / \mathfrak{m})\right)=0$. But this, by Theorem 18.6 in [24], implies that $K_{\mathfrak{m}}=0$ for all $\mathfrak{m}$. Hence $K=0$, and this shows that $f: M \rightarrow N$ is injective.

(iii) $\Rightarrow$ (iv) is clear.

(iv) $\Rightarrow\left(\right.$ i): Let $C$ be an injective cogenerator of $\mathcal{M}_{A}$. By hypothesis, we have that $\mathrm{h}_{N}(C) \rightarrow \mathrm{h}_{M}(C) \rightarrow 0$ is exact and hence $F(C)=0$.

We end the chapter by stating two results which we extended in our first paper.

Theorem 2.2.6. [11, Proposition 3.5] Let $A$ be a local noetherian ring with maximal ideal $\mathfrak{m}$ and residue field $k$. Then, for any coherent functor $F$ and any finitely generated $A$-module $M$, the natural map $\widehat{F(M)} \rightarrow \underset{\lim }{\leftarrow} F\left(M / \mathfrak{m}^{n} M\right)$ is an isomorphism.

Theorem 2.2.7. [11, Proposition 6.1] Let A be a discrete valuation ring (DVR) with parameter $t$. If $F$ is a half exact coherent functor, then $F$ is a direct sum of copies of the identity functor, functors of the form $A / t^{n} \otimes-$, and functors of the form $\operatorname{Hom}_{A}\left(A / t^{n},-\right)$, for various $n \geq 1$. 



\section{Asymptotic stability}

In this chapter, we discuss known results concerning asymptotic associated and attached prime ideals. More details on associated prime ideals and the support of an $A$-module can be found in [1], 44, [21], 24] and [35. For attached prime ideals, see 22 and the appendix to chapter 2 section 6 in 24]. An extensive background to asymptotic stability of sequences of sets of prime ideals and some results can be found in 25 and 30 .

We begin with the definitions of associated and attached prime ideals and describe their main properties. Throughout this chapter, $A$ is a noetherian commutative ring and $F$ is a coherent functor on $\mathcal{M}_{A}$.

\subsection{Associated primes ideals}

Definition 3.1.1. Let $M$ be an $A$-module.

(i) The annihilator of $x \in M$, denoted by $(0: A x)$ or Ann $x$, is the ideal in $A$ defined by

$$
\left(0:{ }_{A} x\right)=\{a \in A: a x=0\} .
$$

(ii) The annihilator of $M$ is the ideal in $A$ defined by

$$
\left(0:{ }_{A} M\right)=\{a \in A: a x=0, \text { for all } x \in M\}
$$

Definition 3.1.2. Let $M$ be an $A$-module. A prime ideal $\mathfrak{p}$ in $A$ is said to be associated to $M$ if $\mathfrak{p}=\left(0:_{A} x\right)$ for some $x \in M$. The set of prime ideals associated to $M$ is denoted by $\operatorname{Ass}_{A} M$. 
Note that, $\mathfrak{p}$ is an associated prime ideal of $M$ if and only if there is an injective homomorphism $A / \mathfrak{p} \rightarrow M$.

Definition 3.1.3. The support of an $A$-module $M$, denoted $\operatorname{Supp}_{A} M$, is the set of primes ideals $\mathfrak{p}$ in $A$ such that $M_{\mathfrak{p}} \neq 0$.

Let $M$ be a finitely generated $A$-module and let $\mathfrak{a}=\left(\begin{array}{lll}0 & :_{A} M\end{array}\right)$. Then $\operatorname{Supp}_{A}(M)=V(\mathfrak{a})$, where $V(\mathfrak{a})$ is the set of all prime ideals containing $\mathfrak{a}$. For ideals $\mathfrak{a}$ and $\mathfrak{b}$, one has $V(\mathfrak{a})=V(\mathfrak{b})$ if and only if $\sqrt{\mathfrak{a}}=\sqrt{\mathfrak{b}}$.

The set of associated prime ideals has the following properties:

(i) If $M$ is a finitely generated $A$-module, then $\operatorname{Ass}_{A} M$ is always a finite set. In fact, $\operatorname{Ass}_{A} M$ consists of the prime ideals which belong to the primary submodules of $M$ in a so called reduced primary decomposition of the zero submodule of $M$, see Theorem 6.8 in [24] or Lemma 7.1.8 in [35].

(ii) For any $A$-module $M, \operatorname{Ass}_{A} M \subset \operatorname{Supp}_{A} M$ and for each $\mathfrak{p} \in \operatorname{Supp}_{A} M$, there is $\mathfrak{q} \in \operatorname{Ass}_{A} M$ such that $\mathfrak{q} \subset \mathfrak{p}$. In particular, the two sets $\operatorname{Ass}_{A} M$ and $\operatorname{Supp}_{A} M$ have the same minimal elements (under inclusion).

(iii) If $M$ is a finitely generated $A$-module and $N$ is any $A$-module, then

$$
\operatorname{Ass}_{A} \operatorname{Hom}_{A}(M, N)=\operatorname{Supp}_{A} M \cap \operatorname{Ass}_{A} N
$$

(see Proposition 10 in section 1.4 of Chapter IV in [4]).

(iv) If $0 \rightarrow M^{\prime} \rightarrow M \rightarrow M^{\prime \prime}(\rightarrow 0)$ is an exact sequence of finitely generated $A$-modules, then we have

$$
\operatorname{Ass}_{A} M^{\prime} \subset \operatorname{Ass}_{A} M \subset \operatorname{Ass}_{A} M^{\prime} \cup \operatorname{Ass}_{A} M^{\prime \prime}
$$

\subsection{Secondary representations and attached prime ideals}

Definition 3.2.1. A nontrivial $A$-module $M$ is said to be secondary if, for each element $a$ in $A$, either $a M=M$ or $a$ is in the radical $\sqrt{\left(0:_{A} M\right)}$ of the annihilator of $M$.

Note that $\mathfrak{p}=\sqrt{\left(0:_{A} M\right)}$ is a prime ideal, and we say that $M$ is $\mathfrak{p}$-secondary.

Proposition 3.2.2. [22, 1.1] All finite direct sums and nontrivial quotients of $\mathfrak{p}$-secondary $A$-modules are $\mathfrak{p}$-secondary.

It follows from 3.2 .2 and the natural map $\bigoplus_{i=1}^{n} M_{i} \rightarrow \sum_{i=1}^{n} M_{i}$ that a finite sum of $\mathfrak{p}$-secondary submodules of an $A$-module is $\mathfrak{p}$-secondary.

Definition 3.2.3. Let $M$ be an $A$-module.

(i) We say that $M$ has a secondary representation if $M$ can be expressed as a finite sum $M=M_{1}+M_{2}+\cdots+M_{n}$ where each $M_{i}$ is a secondary $A-$ submodule of $M$. 
(ii) A secondary representation $M=M_{1}+M_{2}+\cdots+M_{n}$ of $M$ is said to be minimal if the prime ideals $\mathfrak{p}_{i}=\sqrt{\left(0:{ }_{A} M_{i}\right)}, 1 \leq i \leq n$, are all distinct and $M$ cannot be expressed as a sum of a proper sub-collection of the $M_{i}$.

(iii) Given a minimal secondary representation $M=M_{1}+M_{2}+\cdots+M_{n}$ of $M$, the prime ideals $\mathfrak{p}_{i}=\sqrt{\left(0:_{A} M_{i}\right)}, 1 \leq i \leq n$, are called the attached prime

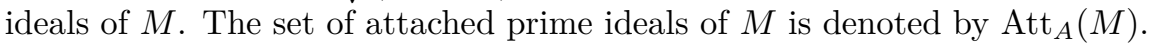

If $M=M_{1}+M_{2}+\cdots+M_{n}$ is a minimal secondary representation of an $A-$ module $M$, the set $\operatorname{Att}_{A} M=\left\{\mathfrak{p}_{1}, \ldots \mathfrak{p}_{n}\right\}$ depends only on $M$. This means that, $\operatorname{Att}_{A} M$ is independent of the chosen secondary representation of $M$.

Definition 3.2.4. A nontrivial $A$-module $M$ is said to be sum-irreducible if any sum of two proper submodules of $M$ is a proper submodule of $M$.

Proposition 3.2.5. [22, 5.1] Any sum-irreducible artinian A-module is a secondary $A-$ module

This Proposition is used to prove

Proposition 3.2.6. [22, 5.2] Every artinian $A$-module has a secondary representation.

Proposition 3.2.7. [22, 2.4 and 4.1] If $0 \rightarrow M^{\prime} \rightarrow M \rightarrow M^{\prime \prime} \rightarrow 0$ is an exact sequence of artinian $A$-modules, then we have

$$
\operatorname{Att}_{A} M^{\prime \prime} \subset \operatorname{Att}_{A} M \subset \operatorname{Att}_{A} M^{\prime} \cup \operatorname{Att}_{A} M^{\prime \prime} .
$$

\subsection{Asymptotic stability of Ass and Att}

A sequence of elements $\left\{x_{n}\right\}_{n=1}^{\infty}$ is said to be asymptotically stable if there is an integer $m$ such that $x_{n}=x_{m}$ for all $n \geq m$, i.e, it is eventually constant.

The topic of asymptotic stability of sequences of sets of prime ideals is classical in commutative algebra. It arose from the following question by Ratliff which appeared in 1976 in his paper [29]:

Question. Let $\mathfrak{p}$ be a prime ideal in a domain $A$. If $\mathfrak{p}$ is in $\operatorname{Ass}_{A}\left(A / \mathfrak{a}^{k}\right)$ for some $k \geq 1$, does it imply that $\mathfrak{p}$ is in $\operatorname{Ass}_{A}\left(A / \mathfrak{a}^{n}\right)$ for all large $n$ ?

In the paper that appeared in 1979, Brodmann gave a counterexample to this question [5, Example 9]. He then posed and answered a related question which then triggered a lot of research in this new direction. He proved

Theorem 3.3.1. Let $\mathfrak{a}$ be an ideal of $A$ and let $M$ be a finitely generated $A_{-}$ module. Then, the two sequences of sets

$$
\left\{\operatorname{Ass}_{A}\left(\mathfrak{a}^{n} M / \mathfrak{a}^{n+1} M\right)\right\}_{n=1}^{\infty} \text { and }\left\{\operatorname{Ass}_{A}\left(M / \mathfrak{a}^{n} M\right)\right\}_{n=1}^{\infty}
$$

are asymptotically stable. 
Many generalizations of 3.3.1 exists in the literature. Rush 32 extended 3.3 .1 by showing that the sequence $\left\{\operatorname{Ass}_{A}\left(M / \mathfrak{a}^{n} N\right)\right\}_{n=1}^{\infty}$ is asymptotically stable, where $M$ is a finitely generated $A$-module and $N$ is a submodule of $M$. In [14, Katz, McAdam and Ratliff Jr. proved that, if $\mathfrak{a}_{1}, \ldots, \mathfrak{a}_{s}$ are ideals of $A$ satisfying some conditions, the sequence $\left\{\operatorname{Ass}_{A}\left(A / \mathfrak{a}_{1}^{g_{n}(1)} \ldots \mathfrak{a}_{s}^{g_{n}(s)}\right)\right\}_{n=1}^{\infty}$ is asymptotically stable. Here $\left\{g_{n}(1), \ldots, g_{n}(s)\right\}_{n=1}^{\infty}$ is an increasing sequence (in the sense $g_{i}(j) \leq g_{i+1}(j)$ ) of $s$-tuples of positive integers.

Also, Kingsbury and Sharp [16] proved that, if $\mathfrak{a}_{1}, \ldots, \mathfrak{a}_{s}$ are ideals of $A$ and $N \subset M$ is a submodule of a finitely generated $A$-module $M$, the sequence $\left\{\operatorname{Ass}_{A}\left(M / \mathfrak{a}_{1}^{g_{n}(1)} \ldots \mathfrak{a}_{s}^{g_{n}(s)} N\right)\right\}_{n=1}^{\infty}$ is asymptotically stable. They also showed that the sequence $\left\{\operatorname{Att}_{A}\left(N^{\prime}:_{N} \mathfrak{a}_{1}^{g_{n}(1)} \ldots \mathfrak{a}_{s}^{g_{n}(s)}\right)\right\}_{n=1}^{\infty}$ is asymptotically stable whenever $N^{\prime} \subset N$ is a submodule of an artinian $A$-module $N$. Again $\left\{g_{n}(1), \ldots, g_{n}(s)\right\}_{n=1}^{\infty}$ is an increasing sequence of $s$-tuples of positive integers. These results are of interest for future research.

Of interest to this thesis are the following theorems:

Theorem 3.3.2. [26. Theorem 1] Let $\mathfrak{a}$ be an ideal of $A$ and $M$ be a finitely generated $A-$ module. Then the sequences of sets

$$
\left\{\operatorname{Ass}_{A} \operatorname{Tor}_{i}^{A}\left(M, \mathfrak{a}^{n} / \mathfrak{a}^{n+1}\right)\right\}_{n=1}^{\infty} \text { and }\left\{\operatorname{Ass}_{A} \operatorname{Tor}_{i}^{A}\left(M, A / \mathfrak{a}^{n}\right)\right\}_{n=1}^{\infty}
$$

are asymptotically stable.

Theorem 3.3.3. [15, Corollary 3.5] Let $M$ and $N$ be finitely generated $A$-modules and $M^{\prime}$ be a submodule of $M$. Then the two sequences of sets

$$
\left\{\operatorname{Ass}_{A}\left(\operatorname{Tor}_{i}^{A}\left(N, M / \mathfrak{a}^{n} M^{\prime}\right)\right)\right\}_{n=1}^{\infty} \text { and }\left\{\operatorname{Ass}_{A}\left(\operatorname{Ext}_{A}^{i}\left(N, M / \mathfrak{a}^{n} M^{\prime}\right)\right)\right\}_{n=1}^{\infty}
$$

are asymptotically stable.

Recently, Se generalized 3.3.1 3.3.2 and 3.3.3 to coherent functors. He proved

Theorem 3.3.4. [33, Theorem 1.11] Let $\mathfrak{a}$ be an ideal of $A, M$ a finitely generated $A-$ module and let $F$ be a coherent functor. Then the two sequences of sets

$$
\left\{\operatorname{Ass}_{A} F\left(\mathfrak{a}^{n} M / \mathfrak{a}^{n+1} M\right)\right\}_{n=1}^{\infty} \text { and }\left\{\operatorname{Ass}_{A} F\left(M / \mathfrak{a}^{n} M\right)\right\}_{n=1}^{\infty}
$$

are asymptotically stable.

In paper B (in part II of our thesis), we have proved Se's result using a different approach.

On the other hand, the theory of attached prime ideals was developed by I.G. Macdonald 222 and others, and is seen to be the dual to the theory of associated prime ideals. The first results concerning asymptotic stability of attached prime ideals appeared in 1986 in the paper by Sharp [34]. He proved

Theorem 3.3.5. Let $\mathfrak{a}$ be an ideal of $A$ and let $N$ be an artinian $A-$ module. Then the two sequences of sets

$$
\left\{\operatorname{Att}_{A}\left(0:_{N} \mathfrak{a}^{n} / 0:_{N} \mathfrak{a}^{n-1}\right)\right\}_{n=1}^{\infty} \text { and }\left\{\operatorname{Att}_{A}\left(0:_{N} \mathfrak{a}^{n}\right)\right\}_{n=1}^{\infty}
$$

are asymptotically stable. 
In the paper that appeared in 1990, Melkersson [27] proved 3.3.5 using a different approach. Rush 32 extended 3.3 .5 by showing that, if $N$ is an artinian $A$-module and $N^{\prime}$ is a submodule of $N$, the sequence of sets of prime ideals $\left\{\operatorname{Att}_{A}\left(N^{\prime}:_{N} \mathfrak{a}^{n}\right)\right\}_{n=1}^{\infty}$ is asymptotically stable. In [26], Melkersson and Schenzel proved that, if $\mathfrak{a}$ is an ideal of $A$ and $N$ an artinian $A$-module, the two sequences of sets

$$
\left\{\operatorname{Att}_{A}\left(\operatorname{Ext}_{A}^{i}\left(\mathfrak{a}^{n} / \mathfrak{a}^{n-1}, N\right)\right)\right\}_{n=1}^{\infty} \text { and }\left\{\operatorname{Att}_{A}\left(\operatorname{Ext}_{A}^{i}\left(A / \mathfrak{a}^{n}, N\right)\right)\right\}_{n=1}^{\infty}
$$

are asymptotically stable. Notice that 3.3 .5 is recovered when $i=0$ in the second assertion.

We have generalized 3.3.5 to coherent functors. See summary of Paper B in chapter 5

\subsection{Depth and grade of a module}

Let $M$ be a finitely generated $A$-module and let $\mathfrak{b}$ be an ideal in $A$ such that $M \neq \mathfrak{b} M$. The depth of $M$ is defined by

$$
\operatorname{depth}_{\mathfrak{b}}(M)=\min \left\{i: \operatorname{Ext}_{A}^{i}(A / \mathfrak{b}, M) \neq 0\right\} .
$$

If $M \neq 0$ and $\mathfrak{b}=(0: A M)$, the grade of $M$ is defined by

$$
\operatorname{grade}(M)=\operatorname{depth}_{\mathfrak{b}} A
$$

i.e, the grade of $M$ is the least $i \geq 0$ such that $\operatorname{Ext}_{A}^{i}(A / \mathfrak{b}, A) \neq 0$.

One can show that

$$
\operatorname{depth}_{\mathfrak{b}}(M)=\min \left\{i: \operatorname{Ext}_{A}^{i}(N, M) \neq 0\right\}
$$

for any finitely generated $A$-module $N$ with $\operatorname{Supp}_{A}(N)=V(\mathfrak{b})$.

Brodmann proved

Theorem 3.4.1. [6, Theorem 2(i)] Let $M$ be a finitely generated $A$-module, and let $\mathfrak{a}$ and $\mathfrak{b}$ be ideals of $A$ such that $M \neq(\mathfrak{a}+\mathfrak{b}) M$. Then the values depth $_{\mathfrak{b}} M / \mathfrak{a}^{n} M$ are independent of $n$ for large $n$.

Se extended this result and proved

Theorem 3.4.2. [33, Theorem 1.11] Let $\mathfrak{a}$ and $\mathfrak{b}$ be ideals of $A, M$ a finitely generated $A$-module and $F$ a coherent functor. Then depth $t_{\mathfrak{b}} F\left(M / \mathfrak{a}^{n} M\right)$ is independent of $n$ for large $n$.

We have given a different proof to 3.4 .2 



\section{Graded modules and Hilbert polynomials}

Most of the material in this chapter can be found in [1], 21], 24], and [35]. Throughout this chapter, $A$ is a commutative ring.

\subsection{Length of a module}

Definition 4.1.1. An $A$-module $M$

(i) is noetherian if, under inclusion, every set of submodules of $M$ has a maximal element.

(ii) is artinian if, under inclusion, every set of submodules of $M$ has a minimal element.

Note that if $M$ is noetherian (respectively artinian), then submodules and quotient modules of $M$ are also noetherian (respectively artinian). Also, $M$ being noetherian is equivalent to every submodule of $M$ being finitely generated. It follows that every finitely generated module over a noetherian ring is noetherian.

Now, let $M$ be a finitely generated $A$-module. Consider a chain of submodules of $M$ of length $n$

$$
M=M_{0} \supset M_{1} \supset \cdots \supset M_{n-1} \supset M_{n}=0 .
$$

The chain 4.1 is a composition series of $M$ if, for $1 \leq i \leq n$, the quotient $M_{i-1} / M_{i}$ is isomorphic to $A / \mathfrak{m}_{\mathfrak{i}}$ for some maximal ideal $\mathfrak{m}_{i}$ in $A$. If $M$ has a composition series of length $n$, then any other composition series of $M$ has length $n$. The length of $M$, denoted by $l_{A}(M)$, is the length of any composition series 
of $M$. Note that, an $A$-module has a composition series if and only if it is both noetherian and artinian, and such a module is called a module of finite length.

Now, given an exact sequence

$$
0 \rightarrow M^{\prime} \rightarrow M \rightarrow M^{\prime \prime} \rightarrow 0
$$

of modules of finite length, we have $l_{A}\left(M^{\prime}\right)-l_{A}(M)+l_{A}\left(M^{\prime \prime}\right)=0$.

We refer the reader to Propositions 6.7, 6.8 and 6.9 in [1] for more details on these facts.

Proposition 4.1.2. Let $A$ be a commutative noetherian ring. Every coherent functor on $\mathcal{M}_{A}$ sends artinian $A$-modules (respectively $A$-modules of finite length) to artinian $A$-modules (respectively $A$-modules of finite length).

Proof. Let $M$ be a finitely generated $A$-module. There is a surjective homomorphism $A^{k} \rightarrow M \rightarrow 0$ for some positive integer $k$. Hence, the sequence

$$
0 \rightarrow \operatorname{Hom}_{A}(M, X) \rightarrow \operatorname{Hom}_{A}\left(A^{k}, X\right) \cong X^{k}
$$

is exact for any $A$-module $X$. Since $X^{k}$ is artinian whenever $X$ is artinian, $\operatorname{Hom}_{A}(M, X)$ is artinian. Now, if $M$ and $N$ are finitely generated $A$-modules such that $h_{N} \rightarrow h_{M} \rightarrow F \rightarrow 0$ is exact, then $F(X)$ is an artinian $A$-module whenever $X$ is artinian. Similarly, $F(X)$ has finite length if $X$ has finite length.

\subsection{Graded and associated graded modules}

A graded ring $R$ is a family $\left(R_{n}\right)_{n \geq 0}$ of subgroups of $R$ such that $R=\bigoplus_{n \geq 0} R_{n}$ and $R_{n} R_{m} \subset R_{n+m}$ for all $n, m \geq 0$. Note that $R_{0}$ is a subring of $R$, and for each $n, R_{n}$ is an $R_{0}$-module. Further, $R$ is noetherian if and only if $R_{0}$ is noetherian and $R$ is finitely generated as an $R_{0}$-algebra. See Proposition 10.7 in [1] or Theorem 13.1 in 24 for details.

Definition 4.2.1. A graded ring $R$ is said to be standard graded or homogeneous if it is generated as an $R_{0}$-algebra by elements of $R_{1}$.

Let $R$ be a graded ring. An $R$-module $M$ is a graded module if $M=\bigoplus_{n \in \mathbb{Z}} M_{n}$ and $R_{n} M_{m} \subset M_{n+m}$ for all $n, m$. Note that $M_{n}$ is an $R_{0}$-module for each $n$. If $R=\bigoplus_{n \geq 0} R_{n}$ is a noetherian graded ring and $M=\bigoplus_{n \in \mathbb{Z}} M_{n}$ is a finitely generated graded $R$-module, then, for each $n, M_{n}$ is finitely generated as an $R_{0}-$ module. In particular, $R_{n}$ is finitely generated as an $R_{0}$-module.

Let $A$ be a ring and $\mathfrak{a}$ an ideal of $A$. The Rees ring of $A$ with respect to $\mathfrak{a}$ is the subring $A[\mathfrak{a} t]=\bigoplus_{n \geq 0} \mathfrak{a}^{n} t^{n}$ of the polynomial ring $A[t]$. If $\mathfrak{a}$ is generated by a finite number of elements in $A$, say $a_{1}, \ldots a_{r}$, the Rees ring is written as $A[\mathfrak{a} t]=A\left[a_{1} t, \ldots a_{r} t\right]$. Thus $A[\mathfrak{a} t]$ is noetherian if $A$ is noetherian. The graded module over the Rees ring $M[\mathfrak{a} t]=\bigoplus_{n \geq 0} \mathfrak{a}^{n} M t^{n}$ is called the Rees module of $M$ with respect to $\mathfrak{a}$. 
Now, let $\mathfrak{a}$ be an ideal in $A$. The associated graded ring of $A$ with respect to $\mathfrak{a}$ is the graded ring $G_{\mathfrak{a}}(A)$ given by $G_{\mathfrak{a}}(A)=\bigoplus_{n>0} \mathfrak{a}^{n} / \mathfrak{a}^{n+1}$. It is a quotient of the Rees ring $A[\mathfrak{a} t]$, namely,

$$
G_{\mathfrak{a}}(A) \cong A[\mathfrak{a} t] / \mathfrak{a} A[\mathfrak{a} t]
$$

If $A$ is noetherian, then $G_{\mathfrak{a}}(A)$ is noetherian.

Let $M$ be an $A$-module. The graded $G_{\mathfrak{a}}(A)$-module

$$
G_{\mathfrak{a}}(M)=\bigoplus_{n \geq 0} \mathfrak{a}^{n} M / \mathfrak{a}^{n+1} M
$$

is called the associated graded module of $M$ with respect to $\mathfrak{a}$.

Now, let $A$ be a noetherian ring, $\mathfrak{a}$ an ideal of $A$ and $G_{\mathfrak{a}}(A)=\bigoplus_{n \geq 0} \mathfrak{a}^{n} / \mathfrak{a}^{n+1}$ the associated graded ring of $A$. For an artinian $A$-module $N$, Kirby [17] introduced the graded module

$$
G^{\mathfrak{a}}(N)=\bigoplus_{n \leq 0}\left(0:_{N} \mathfrak{a}^{-n+1}\right) /\left(0:_{N} \mathfrak{a}^{-n}\right)
$$

dual to the associated graded ring. It is a graded artinian $G_{\mathfrak{a}}(A)$-module and $\left(0:_{N} \mathfrak{a}^{-n+1}\right) /\left(0:_{N} \mathfrak{a}^{-n}\right)$ is an artinian $A$-module for each $n$. See Theorem 1 in [17] and the proof of Theorem 1.2 in [27] for details.

\subsection{Hilbert functions}

An integer-valued polynomial is a polynomial $f \in \mathbb{Q}[X]$ such that $f(n) \in \mathbb{Z}$ for all $n \in \mathbb{Z}$. For example, for each integer $r \geq 0$,

$$
f_{r}(X)=\left(\begin{array}{c}
X+r \\
r
\end{array}\right)=\frac{(X+r)(X+r-1) \ldots(X+1)}{r !}
$$

is an integer-valued polynomial. Every integer-valued polynomial $f$ can be expressed uniquely as

$$
f=\sum_{i=0}^{r} a_{i} f_{i}(X)
$$

where $a_{i} \in \mathbb{Z}, a_{r} \neq 0$ and $r$ is the degree of $f$.

Definition 4.3.1. A function $H: \mathbb{Z} \rightarrow \mathbb{Z}$ is said to be of polynomial type if there is an integer-valued polynomial $P(X)$ (necessarily unique) such that $H(n)=P(n)$ for $n \gg 0$.

Let $R=\bigoplus_{n>0} R_{n}$ be a finitely generated $R_{0}$-algebra such that $R_{0}$ is an artinian ring and let $M=\bigoplus_{n \geq 0} M_{n}$ be a finitely generated graded $R$-module. Then $R_{0}$ is noetherian and $M_{n}$ is a finitely generated $R_{0}$-module. Hence, $l_{R_{0}}\left(M_{n}\right)$ is finite. 
Definition 4.3.2. Let $R$ be a finitely generated $R_{0}$-algebra with $R_{0}$ artinian and let $M=\bigoplus_{n>0} M_{n}$ be a finitely generated graded $R$-module. The Hilbert function $H_{M}: \mathbb{Z} \rightarrow \mathbb{Z}$ of $M$ is given by $H_{M}(n)=l_{R_{0}}\left(M_{n}\right)$.

If $R=R_{0}\left[r_{1}, \ldots, r_{n}\right], r_{i} \in R_{1}$, the Hilbert function $H_{M}$ is of polynomial type, see Theorem 4.1.3 in [7] or Theorem 9.2.1 in [35]. And the polynomial $P_{M}(X)$ such that $H_{M}(n)=P_{M}(n)$ is called the Hilbert polynomial of $M$.

Now, let $\mathfrak{a}$ be an ideal of a noetherian ring $A$ such that $A / \mathfrak{a}$ is an artinian (local) ring and let $M$ be a finitely generated $A$-module. Then $\mathfrak{a}^{n} M / \mathfrak{a}^{n+1} M$ is a finitely generated $A$-module annihilated by $\mathfrak{a}$, hence a finitely generated $A / \mathfrak{a}-$ module. Thus, $l_{A}\left(\mathfrak{a}^{n} M / \mathfrak{a}^{n+1} M\right)$ is finite. Also $l_{A}\left(M / \mathfrak{a}^{n} M\right)$ is finite, see for instance section 9.3 on page 164 in [35]. The Hilbert function $H_{\mathfrak{a}, M}$ with respect to $\mathfrak{a}$ given by $H_{\mathfrak{a}, M}(n)=l_{A}\left(M / \mathfrak{a}^{n} M\right)$ is of polynomial type, see Proposition 11.4 in [1], Theorem 4.1.3 in [7] or Theorem 9.3.1 in [35] for the proof.

Dually, if $N$ is an artinian $A$-module and $0::_{N} \mathfrak{a}$ has finite length, then $0:_{N} \mathfrak{a}^{n}$ has finite length for all $n$. Kirby proved

Theorem 4.3.3. [17, Proposition 2] Let $N$ be an artinian A-module and let $l_{A}\left(0:_{N} \mathfrak{a}\right)$ be finite, then the function $H_{\mathfrak{a}, N}(n)=l_{A}\left(0:_{N} \mathfrak{a}^{n}\right)$ is of polynomial type.

Later, Kodiyalam proved

Theorem 4.3.4. [19, Theorem 2] Let $\mathfrak{a}$ be an ideal of a noetherian ring $A$ and $M, N$ be finitely generated $A$-modules. Then the functions $l_{A}\left(\operatorname{Ext}_{A}^{i}\left(M, N / \mathfrak{a}^{n} N\right)\right)$ and $l_{A}\left(\operatorname{Tor}_{i}^{A}\left(M, N / \mathfrak{a}^{n} N\right)\right)$ are of polynomial type provided $l_{A}\left(M \otimes_{A} N\right)$ is finite.

That $l_{A}\left(M \otimes_{A} N\right)$ is finite means that $\operatorname{Supp}_{A}(M) \cap \operatorname{Supp}_{A}(N)$ consists of finitely many maximal ideals in $A$. To include cases where $l_{A}\left(M \otimes_{A} N\right)$ is not finite, Theoredoscu [36] imposed the condition that

$$
\operatorname{Supp}_{A} M \cap \operatorname{Supp}_{A} N \cap V(\mathfrak{a})
$$

should be a finite set of maximal ideals in $A$. Here, $V(\mathfrak{a})$ is the set of all prime ideals in $A$ containing a. Note that, this condition is equivalent to saying that

$$
\left(M \otimes_{A} N\right) / \mathfrak{a}\left(M \otimes_{A} N\right) \cong M \otimes_{A}(N / \mathfrak{a} N)
$$

has finite length since $\operatorname{Supp}_{A} M \cap \operatorname{Supp}_{A} N \cap V(\mathfrak{a})=\operatorname{Supp}_{A} M \cap \operatorname{Supp}_{A} N / \mathfrak{a} N$.

In this thesis, we have generalized 4.3 .3 and 4.3 .4 to coherent functors, see chapter 5 .

\subsection{Betti and Bass numbers}

In this section, $A$ is a noetherian commutative local ring with maximal ideal $\mathfrak{m}$ and residue field $k$.

Let $M$ be a finitely generated $A$-module. For a non-negative integer $i$, the $i^{\text {th }}$ Betti number of $M$, denoted by $\beta_{i}^{A}(M)$, is given by

$$
\beta_{i}^{A}(M)=l_{A}\left(\operatorname{Tor}_{i}^{A}(k, M)\right)
$$


and the $i^{\text {th }}$ Bass number of $M$, denoted by $\mu_{A}^{i}(M)$, is given by

$$
\mu_{A}^{i}(M)=l_{A}\left(\operatorname{Ext}_{A}^{i}(k, M)\right) .
$$

If $\mathfrak{a}$ is an ideal in $A$, it is known that $l_{A}\left(\mathfrak{a}^{n} / \mathfrak{m} \mathfrak{a}^{n}\right)$ is of polynomial type (see for instance section 3 Theorem 1 in 28$)$. This is equivalent to $\beta_{0}^{A}\left(\mathfrak{a}^{n}\right)=$ $l_{A}\left(\operatorname{Tor}_{0}^{A}\left(k, \mathfrak{a}^{n}\right)\right)$ being of polynomial type via the isomorphism $\mathfrak{a}^{n} / \mathfrak{m} \mathfrak{a}^{n} \cong k \otimes_{A} \mathfrak{a}^{n}$.

In extending this result, Kodiyalam proved

Theorem 4.4.1. [19, Theorem 1] Let $R=\bigoplus_{n>0} R_{n}$ be a homogeneous graded ring with $R_{0}=A$. Let $M=\bigoplus_{n>0} M_{n}$ be a finitely generated $R$-module. Then, for each $i \geq 0$, the Betti numbers $\beta_{i}^{A}\left(M_{n}\right)$ and the Bass numbers $\mu_{A}^{i}\left(M_{n}\right)$ are of polynomial type.

Now, let $\mathfrak{a}$ be an ideal in $A, M$ a finitely generated $A$-module and $R$ the Rees ring of $A$. It follows from 4.4.1 that, for each $i \geq 0$, the Betti numbers $\beta_{i}^{A}\left(\mathfrak{a}^{n} M\right)$ and $\beta_{i}^{A}\left(\mathfrak{a}^{n} M / \mathfrak{a}^{n+1} M\right)$ are of polynomial type. And also, for each $i \geq 0$, the Bass numbers $\mu_{A}^{i}\left(\mathfrak{a}^{n} M\right)$ and $\mu_{A}^{i}\left(\mathfrak{a}^{n} M / \mathfrak{a}^{n+1} M\right)$ are of polynomial type. To cater for modules of the form $M / \mathfrak{a}^{n} M$, Kodiyalam proved

Theorem 4.4.2. [19, Corollary 7] Let $\mathfrak{a}$ be an ideal in A. For any fixed integer $i \geq 0$, both $\beta_{i}^{A}\left(M / \mathfrak{a}^{n} M\right)$ and $\mu_{A}^{i}\left(M / \mathfrak{a}^{n} M\right)$ are of polynomial type.

Note that 4.4 .2 follows from 4.3 .4 with $M=k$. We have made a generalization of 4.4 .2 to coherent functors, see chapter 5

\subsection{Betti numbers related to a half exact coherent functor over a DVR}

In this section, we compute the Betti numbers of $F\left(M / \mathfrak{a}^{n} M\right)$ where $F$ is a half exact coherent functor, $A$ is a discrete valuation ring (DVR) with parameter $t$ and $k=A / \mathfrak{m}$ with $\mathfrak{m}=(t)$. We will show that, for a proper ideal $\mathfrak{a}$ in $A$, the $i^{\text {th }}$ Betti number $\beta_{i}^{A}\left(F\left(M / \mathfrak{a}^{n} M\right)\right)$ is a (constant) polynomial for all $n$.

Since, every non-zero ideal $\mathfrak{a}$ of $A$ is generated by a positive power of $t$, we will consider $\mathfrak{a}=(t)$. Throughout this section, $t^{r}$ will denote the ideal $\left(t^{r}\right)$.

Now, every finitely generated module over a DVR is a direct sum of copies of $A$ and modules of the form $A / t^{m}$ for various $m \geq 1$. We will consider these cases. Let $M=A / t^{m}$, then

$$
\begin{aligned}
M / t^{n} M & =\left(A / t^{m}\right) /\left(t^{n}\left(A / t^{m}\right)\right) \\
\cong\left(A / t^{m}\right) /\left(\left(t^{n}, t^{m}\right) / t^{m}\right) & \cong A /\left(t^{n}, t^{m}\right) \\
& = \begin{cases}A / t^{n}, & 1 \leq n \leq m \\
A / t^{m}, & n \geq m\end{cases}
\end{aligned}
$$

Now, consider the exact sequence

$$
0 \rightarrow A \rightarrow A \rightarrow k \rightarrow 0
$$


where $A \rightarrow A$ is multiplication by $t, k=A / t$ and $A \rightarrow k$ is the natural map. Then, for any $A$-module $M$, we have an exact sequence

$$
0 \rightarrow 0 \rightarrow \operatorname{Tor}_{1}^{A}(k, M) \rightarrow M \rightarrow M \rightarrow k \otimes_{A} M \rightarrow 0
$$

where $M \rightarrow M$ is multiplication by $t$. Thus

$$
\operatorname{Tor}_{i}^{A}(k, M)= \begin{cases}k \otimes_{A} M, & i=0 \\ 0:_{M} t, & i=1 \\ 0, & i>1\end{cases}
$$

If $M=A / t^{n}$ for $n \geq 1$, we get

$$
\begin{aligned}
\operatorname{Tor}_{0}^{A}\left(k, A / t^{n}\right) & \cong A / t \otimes_{A} A / t^{n} \\
& \cong\left(A / t^{n}\right) /\left(t\left(A / t^{n}\right)\right) \\
& \cong A /\left(t, t^{n}\right) \\
& =A / t \\
& =k
\end{aligned}
$$

and

$$
\begin{aligned}
\operatorname{Tor}_{1}^{A}\left(k, A / t^{n}\right) & =0:_{A / t^{n}} t \\
& \cong\left(t^{n}:_{A} t\right) / t^{n} \\
& \cong t^{n-1} / t^{n} \\
& \cong k .
\end{aligned}
$$

From 4.3, 4.4 and 4.5 we conclude that, for $n \geq 1$,

$$
\operatorname{Tor}_{i}^{A}\left(k, A / t^{n}\right)= \begin{cases}k, & i=0,1 \\ 0, & i>1\end{cases}
$$

Now, let $F$ be a half exact coherent functor over a DVR $A$ with parameter $t$. Since every half exact coherent functor over a DVR is a direct sum of copies of the identity functor, functors of the form $A / t^{s} \otimes_{A}-$ and of the form $\operatorname{Hom}_{A}\left(A / t^{s},-\right)$ for various $s \geq 1$ (see Theorem 2.2.7), we consider each of these cases separately.

Case 1 Suppose that $F$ is the identity functor.

i) If $M=A$, we have by 4.6 that

$$
\operatorname{Tor}_{i}^{A}\left(k, F\left(M / t^{n} M\right)\right)=\operatorname{Tor}_{i}^{A}\left(k, A / t^{n}\right)= \begin{cases}k, & i=0,1 \\ 0, & i>1\end{cases}
$$

ii) If $M=A / t^{m}$, then

$$
\begin{aligned}
\operatorname{Tor}_{i}^{A}\left(k, F\left(M / t^{n} M\right)\right) & =\operatorname{Tor}_{i}^{A}\left(k,\left(A / t^{m}\right) /\left(t^{n}\left(A / t^{m}\right)\right)\right. \\
& \cong \begin{cases}\operatorname{Tor}_{i}^{A}\left(k, A / t^{n}\right), & 1 \leq n \leq m \\
\operatorname{Tor}_{i}^{A}\left(k, A / t^{m}\right), & n \geq m\end{cases}
\end{aligned}
$$


where the isomorphism is due to 4.2 . Thus, in any case, we have by 4.6 that

$$
\operatorname{Tor}_{i}^{A}\left(k,\left(A / t^{m}\right) /\left(t^{n}\left(A / t^{m}\right)\right)= \begin{cases}k, & i=0,1 \\ 0, & i>1\end{cases}\right.
$$

Case 2 Suppose that $F=A / t^{s} \otimes_{A}-$, for $s \geq 1$.

i) If $M=A$, we have

$$
\begin{aligned}
F\left(M / t^{n} M\right) & =A / t^{s} \otimes_{A} A / t^{n} \\
& \cong A /\left(t^{n}, t^{s}\right) \\
& = \begin{cases}A / t^{s}, & 1 \leq s \leq n \\
A / t^{n}, & s \geq n\end{cases}
\end{aligned}
$$

Hence, 4.6 shows that

$$
\operatorname{Tor}_{i}^{A}\left(k, A / t^{s} \otimes_{A} A / t^{n}\right)= \begin{cases}k, & i=0,1 \\ 0, & i>1\end{cases}
$$

ii) If $M=A / t^{m}$, by using 4.2 followed by (4.8), we get

$$
\begin{aligned}
F\left(M / t^{n} M\right) & =A / t^{s} \otimes_{A}\left(\left(A / t^{m}\right) / t^{n}\left(A / t^{m}\right)\right) \\
& \cong \begin{cases}A / t^{s} \otimes_{A} A / t^{n}, \quad 1 \leq n \leq m \\
A / t^{s} \otimes_{A} A / t^{m}, \quad n \geq m\end{cases} \\
& \cong \begin{cases}A / t^{s}, & 1 \leq s \leq n \\
A / t^{n}, & n \leq s \leq m \\
A / t^{m}, & s \geq m\end{cases}
\end{aligned}
$$

Again by 4.6 we have

$$
\operatorname{Tor}_{i}^{A}\left(k, A / t^{s} \otimes_{A}\left(\left(A / t^{m}\right) / t^{n}\left(A / t^{m}\right)\right)= \begin{cases}k, & i=0,1 \\ 0, & i>1\end{cases}\right.
$$

Case 3 Suppose that $F=\operatorname{Hom}_{A}\left(A / t^{s},-\right)$, for $s \geq 1$.

i) If $M=A$, we have

$$
\begin{aligned}
F\left(M / t^{n} M\right) & =\operatorname{Hom}_{A}\left(A / t^{s}, A / t^{n}\right) \\
& \cong 0:_{A} / t^{n} t^{s} \\
& =\left(t^{n}:_{A} t^{s}\right) / t^{n} \\
& \cong \begin{cases}t^{n-s} / t^{n}, & 1 \leq s \leq n \\
A / t^{n}, & s \geq n\end{cases} \\
& \cong \begin{cases}A / t^{s}, & 1 \leq s \leq n \\
A / t^{n}, & s \geq n\end{cases}
\end{aligned}
$$


and again by 4.6 we get

$$
\operatorname{Tor}_{i}^{A}\left(k, \operatorname{Hom}_{A}\left(A / t^{s}, A / t^{n}\right)\right)= \begin{cases}k, & i=0,1 \\ 0, & i>1\end{cases}
$$

ii) If $M=A / t^{m}$, by using 4.2 followed by 4.9 , we have

$$
\begin{aligned}
F\left(M / t^{n} M\right) & =\operatorname{Hom}_{A}\left(A / t^{s},\left(A / t^{m}\right) / t^{n}\left(A / t^{m}\right)\right) \\
& \cong \begin{cases}\operatorname{Hom}_{A}\left(A / t^{s}, A / t^{n}\right), & 1 \leq n \leq m \\
\operatorname{Hom}_{A}\left(A / t^{s}, A / t^{m}\right), & n \geq m\end{cases} \\
& \cong \begin{cases}A / t^{s}, & 1 \leq s \leq n \\
A / t^{m}, & n \leq s \leq m \\
A / t^{n}, & s \geq m\end{cases}
\end{aligned}
$$

Thus, in this case also, 4.6 shows that

$$
\operatorname{Tor}_{i}^{A}\left(k, \operatorname{Hom}_{A}\left(A / t^{s}, A / t^{n}\right)\right)= \begin{cases}k, & i=0,1 \\ 0, & i>1\end{cases}
$$

Therefore, the $i^{\text {th }}$ Betti number $\beta_{i}^{A}\left(F\left(M / \mathfrak{a}^{n} M\right)\right)$ is a (constant) polynomial for all large $n$. 


\section{An overview of the papers}

This chapter gives an overview of the papers that form part of this thesis.

\subsection{Summary of Paper A}

The main aim of this paper is to extend Hartshorne's result 2.2.7 to Dedekind domains. That is, to characterize half-exact coherent functors over principal ideal domains (PIDs) and more generally over Dedekind domains. Our proof has similar steps as Hartshorne's proof. Throughout, we assume that $A$ is a noetherian commutative ring and $F$ is a coherent functor over the category $\mathcal{M}_{A}$.

Now, for any ideal $\mathfrak{a}$ in $A$ and any finitely generated $A$-module $M$, there is a natural map

$$
\widehat{F(M)} \rightarrow \underset{\lim }{\longleftarrow} F\left(M / \mathfrak{a}^{n} M\right)
$$

which arises by taking the inverse limit of $F(M) / \mathfrak{a}^{n} F(M) \rightarrow F\left(M / \mathfrak{a}^{n} M\right)$. Here $\widehat{F(M)}$ is the completion of $F(M)$ in the $\mathfrak{a}$-adic topology. In section 2 , we show that this natural map is an isomorphism whenever $\operatorname{Supp}(M / \mathfrak{a} M)$ consists of only maximal ideals of $A$. This generalizes 2.2.6 The condition on the support is equivalent to saying that $M / \mathfrak{a} M$ has finite length. To prove the result, we make use of the Mittag-Lefler condition (see section 1.5 to obtain an exact sequence

$$
\lim _{Y} \mathrm{~h}_{Y}\left(M / \mathfrak{a}^{n} M\right) \rightarrow \lim _{\longleftarrow} \mathrm{h}_{X}\left(M / \mathfrak{a}^{n} M\right) \rightarrow \lim _{\longleftarrow} F\left(M / \mathfrak{a}^{n} M\right) \rightarrow 0
$$

where $X, Y$ are finitely generated $A$-modules such that $\mathrm{h}_{Y} \rightarrow \mathrm{h}_{X} \rightarrow F \rightarrow 0$ is exact. We then show that

$$
\lim _{\longleftarrow} \mathrm{h}_{N}\left(M / \mathfrak{a}^{n} M\right) \cong \widehat{\mathrm{h}_{N}(M)}
$$


for any finitely generated $A$-module $N$, and use an appropriate commutative diagram and 1.3 .5 to complete the proof.

In section 3 , we devote ourselves to the construction of a functor $\tilde{F}$ which also appears in [11] in a more general setting. For a multiplicative subset $S \subset A$ and a coherent functor $F$, we use the fact that every finitely generated $S^{-1} A$-module $M$ is isomorphic to $S^{-1} N$ for some finitely generated $A$-module $N$ to define $\tilde{F}$ on finitely generated $S^{-1} A$-modules. We then show that $\tilde{F}$ is left-, right- or halfexact whenever $F$ is left-, right- or half-exact. It also turns out that $\tilde{F}$ is coherent whenever $F$ is. When $S=A \backslash \mathfrak{p}$, we denote $\tilde{F}$ by $F_{\mathfrak{p}}$ and we use this in the proof of our main theorem.

In section 4 , we prove our main results. For any $A$-linear functor $F$, we consider the exact sequence

$$
F(A) \otimes-\stackrel{\alpha}{\longrightarrow} F \longrightarrow F_{0} \longrightarrow 0
$$

(see section 2.2). We first show that $\alpha_{M}: F(A) \otimes M \rightarrow F(M)$ is injective for any finitely generated $A$-module $M$ with projective dimension at most 1 . We then show that if $F$ is a half exact coherent functor over a PID, then $F_{0}$ is left exact and the sequence (5.1) splits, i.e, $F \cong F(A) \otimes-\oplus F_{0}$. From this, we deduce that $F$ is a direct sum of copies of the identity functor, functors of the form $\operatorname{Hom}_{A}\left(A / p^{s},-\right)$ and functors of the form $A / p^{s} \otimes-$ for various $s \geq 1$, where $p$ is an irreducible element in $A$.

When $A$ is a Dedekind domain, by localizing to prime ideals, we show that $F_{0}$ is left exact and hence the sequence (5.1) splits. We again deduce from this that $F$ is a direct sum of functors of the form $\operatorname{Hom}_{A}(P,-), \operatorname{Hom}_{A}\left(A / \mathfrak{p}^{s},-\right)$ and $A / \mathfrak{p}^{s} \otimes-$ for various $s \geq 1$, where $P$ is a finitely generated projective $A$-module and $\mathfrak{p}$ is a prime ideal in $A$.

\subsection{Summary of Paper B}

In this paper we study asymptotic stability of sets of prime ideals and Hilbert polynomials of graded modules related to coherent functors. Throughout the paper, we assume that $A$ is a commutative noetherian ring and $R=A\left[a_{1}, \ldots, a_{r}\right]$ is a graded $A$-algebra where $a_{i}$ is an element of degree 1 in $R$.

In section 2 , we first show that if $\mathcal{S}$ is a thick subcategory (i.e, is closed under kernels, cokernels and extensions) of the category of graded $R$-modules, then for each coherent functor $F$ and every $U$ in $\mathcal{S}$, the graded $R$-module $F(U)$ is also in $\mathcal{S}$. We then show that if $\mathcal{S}$ is a Serre subcategory (i.e, is closed under subobjects, quotients and extensions) of the category of graded $R$-modules and $f: U \rightarrow V$ is a graded $R$-homomorphism, then $\operatorname{Ker} F(f)$ and Coker $F(f)$ are in $\mathcal{S}$ whenever Ker $f$ and Coker $f$ are in $\mathcal{S}$. This is true in particular for the category of finitely generated modules and the category of artinian modules.

Next, we give a different proof to 3.3.4 We then end the section by extending Sharp's result 3.3.5 We prove that, if $N$ is an artinian $A$-module, $\mathfrak{a}$ an ideal of $A$ and $F$ a coherent functor, the two sequences $\left\{\operatorname{Att}_{A} F\left(\left(0:_{N} \mathfrak{a}^{n}\right) /\left(0:_{N} \mathfrak{a}^{n-1}\right)\right)\right\}_{n=1}^{\infty}$ and $\left\{\operatorname{Att}_{A} F\left(0:_{N} \mathfrak{a}^{n}\right)\right\}_{n=1}^{\infty}$ are asymptotically stable. We obtain the first assertion 
by using the fact that $G^{\mathfrak{a}}(N)=\bigoplus_{n<0}\left(0:_{N} \mathfrak{a}^{-n+1}\right) /\left(0:_{N} \mathfrak{a}^{-n}\right)$ is an artinian $G(A)$-module (see section 4.2). Hence, we have that $F\left(G^{\mathfrak{a}}(N)\right.$ ) is an artinian $G(A)$-module and Theorem 3.1 in 34 gives the result.

For the second assertion, we applying $F$ to the exact sequence

$$
0 \longrightarrow U \stackrel{f}{\longrightarrow} V \longrightarrow W \longrightarrow 0
$$

where $U, V$ and $W$ are graded modules over the Rees ring $R=A[\mathfrak{a} t]$ which in degree $-n, n=1,2,3, \ldots$, are defined by $U_{-n}=0:_{N} \mathfrak{a}^{n-1}, V_{-n}=0:_{N} \mathfrak{a}^{n}$ and $W_{-n}=\left(0::_{N} \mathfrak{a}^{n}\right) /\left(0::_{N} \mathfrak{a}^{n-1}\right)$. Taking $C=$ Coker $F(f)$, we consider the exact sequence in degree $-n$

$$
F\left(0::_{N} \mathfrak{a}^{n-1}\right) \longrightarrow F\left(0:_{N} \mathfrak{a}^{n}\right) \longrightarrow C_{-n} \longrightarrow 0
$$

Since $C$ will be an artinian graded $R$-module, $\operatorname{Att}_{A}\left(C_{-n}\right)$ is asymptotically stable. The result now follows from the inclusions

$$
\operatorname{Att}_{A} C_{-n} \subset \operatorname{Att}_{A} F\left(0:_{N} \mathfrak{a}^{n}\right) \subset \operatorname{Att}_{A} F\left(0:_{N} \mathfrak{a}^{n-1}\right) \cup \operatorname{Att}_{A}\left(C_{-n}\right)
$$

and the fact that the sets in the above inclusions are finite.

In section 3, we study Hilbert polynomials related to coherent functors. We obtain two results that extends Kirby's result 4.3.3. In the first, we show that if $N$ is an artinian $A$-module and $0::_{N} \mathfrak{a}^{n}$ has finite length, then the length of $F\left(0::_{N} \mathfrak{a}^{n}\right)$ is of polynomial type (see section 4.3 for the definition). We achieve this by first showing that the length of $\operatorname{Hom}_{A}\left(X, 0:_{N} \mathfrak{a}^{n}\right)$ is of polynomial type when $X$ is a finitely generated $A$-module. Letting $Z$ to be the cokernel of the map $X \rightarrow Y$ such that $\mathrm{h}_{Y} \rightarrow \mathrm{h}_{X} \rightarrow F \rightarrow 0$ is exact, we obtain the exact sequence

$$
0 \rightarrow \mathrm{h}_{Z}\left(0:_{N} \mathfrak{a}^{n}\right) \rightarrow \mathrm{h}_{Y}\left(0:_{N} \mathfrak{a}^{n}\right) \rightarrow \mathrm{h}_{X}\left(0:_{N} \mathfrak{a}^{n}\right) \rightarrow F\left(0:_{N} \mathfrak{a}^{n}\right) \rightarrow 0
$$

The result now follows by the additive property of the length function.

In the second result, we assume that $F\left(0:_{N} \mathfrak{a}^{n}\right)$ has finite length for all $n$, and prove that the length of $F\left(0:_{N} \mathfrak{a}^{n}\right)$ is of polynomial type. We also prove its dual that, if $M$ is a finitely generated $A$-module and $F\left(M / \mathfrak{a}^{n} M\right)$ has finite length for all $n$, then $F\left(M / \mathfrak{a}^{n} M\right)$ is of polynomial type.

Next, we show that the composite of two coherent functors is coherent. We then use this to prove two results. The first is Se's result 3.4.2. We also show that grade $F\left(M / \mathfrak{a}^{n} M\right)$ is independent of $n$ for large $n$. The second is an extension of Kodiyalam's result 4.4.1. We show that, if $A$ is local, the $i^{\text {th }}$ Betti number and the $i^{\text {th }}$ Bass number of $F\left(M / \mathfrak{a}^{n} M\right)$ are of polynomial type. 


\section{Bibliography}

[1] Atiyah M. F. and MacDonald I. G., Introduction to commutative algebra. Addison-Wesley, 1969.

[2] Auslander M., Coherent functors. Proc. Conf. Categorical Algebra, Springer, New York (1966), 189-231.

[3] Bourbaki N., Algebra I, Chapters 1-3. Springer, 1989.

[4] Bourbaki N., Commutative algebra, Chapters 1-7. Springer, 1989.

[5] Brodmann M, Asymptotic stability of Ass $\left(\frac{M}{I^{n} M}\right)$. Proc. Amer. Math. Soc. 74 (1) (1979), 16-18.

[6] Brodmann M, The asymptotic nature of the analytic spread. Math. Proc. Camb. Phil. Soc. 86 (1) (1979), 35-39.

[7] Bruns W. and Herzog J., Cohen-Macaulay rings. Revised edition. Camb. Univ. Press, 1993.

[8] Cartan H. and Eilenberg S., Homological algebra. Princeton University Press, 1956.

[9] Dummit D. S. and Foote R. M., Abstract algebra. Third edition. John Wiley and sons, Inc, 2004.

[10] Freyd P., Abelian categories: An introduction to the theory of functors. Harper and Row, 1964.

[11] Hartshorne R., Coherent functors. Advances in Mathematics 140 (1998), 4494. 
[12] Hilton P. J. and Stammbach U., A course in homological algebra. Second edition. Springer, 1997.

[13] Kashiwara M. and Schapira P., Categories and sheaves. Springer, 2006.

[14] Katz D., McAdam S. and Ratliff L. J. Jr., Prime divisors and divisoral ideals. J. Pure Appl. Algebra 59 (1989), 179-186.

[15] Katz D. and West E., A linear function associated to asymptotic prime divisors. Proc. Amer. Math. Soc. 132 (6) (2003), 1589-1597.

[16] Kingsbury A. K. and Sharp R. Y., Asymptotic behaviour of certain sets of prime ideals. Proc. Amer. Math. Soc. 124 (6) (1996), 1703-1711.

[17] Kirby D. Artinian modules and Hilbert polynomials. Quart. J. Math. Oxford Ser. 24 (2) (1973), 47-57.

[18] Kirby D., Hilbert functions and the extension functor. Math. Camb. Proc. Phil. Soc. 105 (3) (1989), 441-446.

[19] Kodiyalam V., Homological invariants of powers of an ideal. Proc. Amer. Math. Soc. 118 (3) (1993), 757-764.

[20] Lam T. Y., Lectures on modules and rings. Springer, 1999.

[21] Lang S., Algebra. Revised third edition. Springer, 2002.

[22] Macdonald I. G., Secondary representations of modules over a commutative ring. Symposia Mathematica 11 (1973), 23-43.

[23] Mac Lane S., Categories for the working mathematician. Second edition. Springer, 1998.

[24] Matsumura H., Commutative ring theory. Camb. Univ. Press, 1986.

[25] McAdam S., Asymptotic prime divisors. Lecture notes in Mathematics $\mathbf{1 0 2 3}$ (Springer, 1983).

[26] Melkersson L. and Schenzel P., Asymptotic prime ideals related to derived functors. Proc. Amer. Math. Soc. 117 (4) (1993), 935-938.

[27] Melkersson L., On asymptotic stability for sets of prime ideals connected with the powers of an ideal. Math. Proc. Camb. Phil. Soc. 107 (1990), 267-271.

[28] Northcott D. G. and Rees D., Reductions of ideals in local rings. Proc. Camb. Phil. Soc. 50 (1954), 145-158.

[29] Ratliff L. J. Jr., On prime divisors of $I^{n}, n$ large. Michigan Math. J. 23 (1976), $337-352$.

[30] Ratliff L. J. Jr., A brief survey and history of asymptotic prime divisors. Rocky Mountain J. of Math. 13 (3) (1983), 437-459. 
[31] Rotman J.J., An introduction to homological algebra. Second edition. Springer, 2009.

[32] Rush D.E., Asymptotic primes and integral closure in modules. Quart. J. Math. Oxford Ser. 43 (172) (1992), 477-499.

[33] Se T., Covariant functors and asymptotic stability. J. Algebra 484 (2017), 247-264.

[34] Sharp R. Y., Asymptotic behaviour of certain sets of attached prime ideals. J. Lond. Math. Soc 34 (2) (1986) 212-218.

[35] Singh B., Basic commutative algebra. World Scientific Publishing Co. Pte. Ltd., 2011.

[36] Theoredoscu E., Derived functors and Hilbert polynomials. Math. Proc. Camb. Phil. Soc. 132 (1) (2002), 75-88.

[37] Weibel C. A., An introduction to homological algebra. Camb. Univ. Press, 1994. 


\section{Part II}

\section{Included Papers}





\section{Papers}

The papers associated with this thesis have been removed for copyright reasons. For more details about these see:

http://urn.kb.se/resolve?urn=urn:nbn:se:liu:diva-156730 



\section{INSTITUTE OF TECHNOLOGY}

Linköping Studies in Science and Technology, Dissertation No. 1982, 2019

Department of Mathematics (MAI)

Linköping University

SE-581 83 Linköping, Sweden

www.liu.se 